Article

\title{
Numerical Study on the Influence of Mass and Stiffness Ratios on the Vortex Induced Motion of an Elastically Mounted Cylinder for Harnessing Power
}

\author{
Vidya Chandran ${ }^{1}$, Sekar M. ${ }^{2}$, Sheeja Janardhanan ${ }^{3, *}$ and Varun Menon ${ }^{4}$ (i) \\ 1 Department of Mechanical Engineering, Karunya Institute of Technology and Sciences, Coimbatore, \\ Tamil Nadu 600018, India; vidya.rudn@gmail.com \\ 2 Department of Mechanical Engineering, AAA College of Engineering and Technology, Sivakasi, \\ Tamil Nadu 600018, India; mailtosekar@gmail.com \\ 3 Department of Mechanical Engineering, SCMS School of Engineering and Technology, Ernakulam, \\ Kerala 673307, India \\ 4 Department of Computer Science and Engineering, SCMS School of Engineering and Technology, \\ Ernakulam, Kerala 673307, India; varungmenon46@gmail.com \\ * Correspondence: sheejajanardhanan@scmsgroup.org; Tel.: +91-828-194-3531
}

Received: 10 September 2018; Accepted: 25 September 2018; Published: 27 September 2018

\begin{abstract}
Harnessing the power of vortices shed in the wake of bluff bodies is indeed a boon to society in the face of fuel crisis. This fact serves as an impetus to develop a device called a hydro vortex power generator (HVPG), comprised of an elastically mounted cylinder that is free to oscillate in the cross-flow (CF) direction even in a low velocity flow field. The oscillatory motions in turn can be converted to useful power. This paper addresses the influence of system characteristics viz. stiffness ratio $\left(k^{*}\right)$ and mass ratio $\left(m^{*}\right)$ on the maximum response amplitude of the elastically mounted cylinder. Computational fluid dynamics (CFD) simulations have been used here to solve a two way fluid-structure interaction (FSI) problem for predicting the trend of variation of the non-dimensional amplitude $Y / D$ with reduced velocity $U_{r}$ through a series of simulations. Maximum amplitude motions have been attributed to the lowest value of $\mathrm{m}^{*}$ with $U_{r}=8$. However, the maximum lift forces correspond to $U_{r}=4$, providing strong design inputs as well as indicating the best operating conditions. The numerical results have been compared with those of field tests in an irrigation canal and have shown reasonable agreement.
\end{abstract}

Keywords: computational fluid dynamics (CFD); flow around cylinder; fluid structure interaction (FSI); hydrodynamic response; numerical methods; simulation and modeling; vortex induced vibration (VIV) ratio

\section{Introduction}

As the sources of fossil fuels are depleting at a faster pace, energy scientists all over the world are keen on the search for new technologies that can provide renewable and clean energy. Hydroelectric power generation is of course a clean source of energy, but considering the capital investment and the effects of dams on natural ecosystems, the need for a much cleaner energy source becomes more important. This paper discusses the design and manufacture of the hydro vortex power generator (HVPG) model, which when scaled up can be viewed as one such cleaner source of electricity. Also, the paper discusses a numerical method to optimize the design parameters of HVPG model. The principle behind the working of HVPG is vortex shedding in the wake of bluff bodies in fluid flow. The phenomenon of vortex shedding behind bluff bodies has been an extensively researched topic $[1,2]$. The presence of such vortex shedding has been considered undesirable and researchers had been in 
search of methods to suppress vortex shedding [3]. Vortex power was proved useful to mankind by the researchers at Michigan University, who first converted vortex power into electricity [4,5]. HVPG works on the principle of vortex induced vibration of bluff bodies subjected to fluid current. The power of vortices shed in the wake of these bluff bodies are converted into vibration energy and then into electricity. HVPG can be made useful as a single standing power unit that can provide electricity to remote locations; and also as a multiunit module which can supply power to the grid [6].The paper discusses the design optimization of a single, power-generating, scaled-down module, harnessing power from vortices. In this paper, an attempt has been made to optimize the design based on the major influencing parameters, oscillating mass ratio $\left(m^{*}\right)$ and stiffness ratio $\left(k^{*}\right)$. Griffin consolidated experimental results and plotted them to show the dependence of maximum amplitude on system characteristics [7]. However, the drawback of Griffin's plot was its considerable scatter which could be attributed to the inclusion of mode shapes also as an influencing parameter. Later researchers could successfully reduce the scatter in Griffin's plot and establish a simple relationship between maximum amplitude and mass damping parameter by eliminating mode shapes from the list of variables $[7,8]$. The simplified mass damping parameter as in [8] was applicable for cylinders of high and low mass ratios equally. Many researchers have formulated empirical formulae to express the parametric relationship and experimentally verified the correlations $[9,10]$. Later in the experiments, however, it has been observed that with variation in the damping ratio, $U_{r}$ and the Strouhal number $(S t)$ varies [11]. Also, these experiments revealed relatively larger response amplitude compared to other studies. Recently researchers have also succeeded in theoretically proving the effectiveness of harnessing VIV energy for powering underwater mooring platforms [12].

The present work studies the influence of $k^{*}$ and $m^{*}$ on maximum response amplitude of an elastically mounted cylinder with a single DOF. It also provides a detailed insight into the vortex shedding pattern at various $U_{r}$. The simulations are carried out at Re of the order $10^{4}$ which corresponds to the realistic flow regime encountered by power generating vortices [13]. The numerical results have been verified using field tests conducted at Palissery irrigation canal (Palissery irrigational canal is one of the irrigation projects by Government of Kerala located in Thrissur district, Kerala, India).

Previous studies in the same domain had only considered the effect of complex and coupled parameters such as the Skop Griffin parameter [14]. The novelty in the present work is the effort made to represent the influence of tangible parameters viz the stiffness coefficient and mass of the cylinder. These parameters are easily controllable from a designer's point of view. A non-dimensional approach has been used here to generalize the results for the design of power harnessing devices of any scale.

\section{The Concept of HVPG}

HVPG works on the principle of vortex induced vibration (VIV). If a bluff body is not completely secured with at least one degree of freedom motion, and the frequency of vortex shedding matches the natural frequency of the structure, the structure begins to resonate, vibrating with harmonic oscillations of large amplitude. This phenomenon is known as 'lock-in'. During lock-in, vortex shedding frequency shifts to the natural frequency of the structure, leading to large amplitude vibrations. Vortex shedding in the wake of a cylinder is shown in Figure 1.

The vortex shedding occurs at a discrete frequency and is a function of the Reynolds number $(R e)$, defined by Equation (1)

$$
R e=\frac{\rho V D}{\mu}
$$

The dimensionless frequency of the vortex shedding, $S t=f_{v} D / V$, is approximately equal to 0.2 when the Reynolds number is greater than 1000 [15]. When vortices are shed from the cylinder, uneven pressure distribution develops around the upper and lower surfaces of the cylinder, generating an 
oscillating hydrodynamic lift force on the cylinder. This unsteady force given by Equation (2) can induce significant cross flow vibrations on a structure, especially if the resonance condition is met.

$$
F_{L}=C_{L} \frac{1}{2} \rho A V^{2}
$$

where $F_{L}$ is the lift force and $C_{L}$ is the coefficient of lift. $\rho$ is the density of water, A the projected area in the direction of flow, and $\mathrm{V}$ is the velocity of flowing water. The cylinder also experiences a net force along the flow direction and is called the drag force and is given by the Equation (3).

$$
F_{D}=C_{D} \frac{1}{2} \rho A V^{2}
$$

where $F_{D}$ is the drag force and $C_{D}$ is the drag coefficient.

The oscillating lift force acting on the cylinder makes the cylinder oscillate in the cross flow (CF) direction at the frequency of vortex shedding. For the making of HVPG, the cylinder has been mounted elastically, which enables the entire module to be considered as a spring-mass system with the cylinder considered as the mass and the elastic supports as springs. When the natural frequency of spring-mass system matches the vortex shedding frequency, the cylinder oscillates with large amplitudes. The linear motion of the mass can then be converted to rotary motions through a slider-crank mechanism and the crank rotations can be used to drive a generator unit.

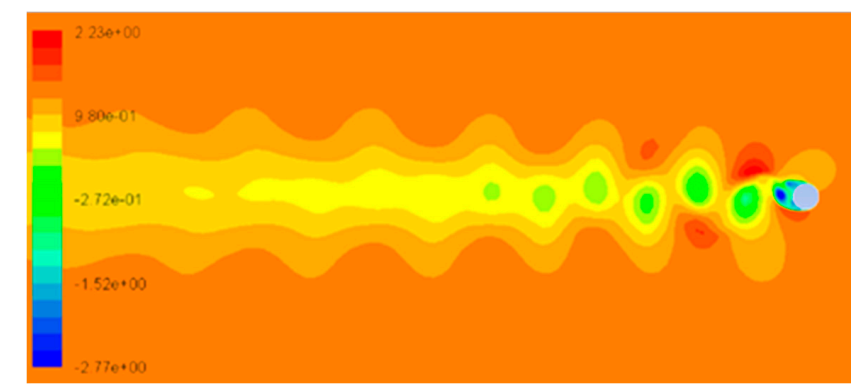

Figure 1. Shedding of alternate vortices behind a cylinder represented as pressure $\left(\mathrm{N} / \mathrm{m}^{2}\right)$ contours. (Von Kármán Vortex Street).

\section{Mathematical Model}

A single power module of HVPG has been modeled as a spring mass system undergoing instability induced vibration. The instability is caused by the shedding of vortices in the wake of the cylinder when the flow encounters a bluff body. Alternate vortex shedding causes oscillatory forces that induce structural vibrations, where the rigid cylinder is now similar to a spring-mass system with a harmonic forcing term. This phenomenon is referred to as heave motion [14]. Equation of motion [16] for this system can be written as

$$
m \ddot{Y}+c \dot{Y}+k Y=F(t)
$$

where $Y$ is the displacement of the cylinder under VIV in the cross-flow direction; $m$ is the sum of mass of the oscillating system or body mass, $m_{b}$ and added mass of the system, $m_{a} . m_{a}$ is defined as $m_{a}=C_{A} m_{b}$, where $C_{A}$ is the added mass coefficient. $c$ is the damping coefficient and $k$ is the coefficient of stiffness of the spring mass system. $F(t)$ is the time varying force acting on the cylinder due the flow instability. Relatively small oscillation amplitudes are approximated by

$$
F(t)=F_{L} \sin \left(\omega_{v} t+\varphi\right)
$$

where $\omega_{v}$ is the circular frequency of vortex shedding and $\varphi$ the phase difference between the force and cylinder displacement. $F_{L}$ is the maximum value of oscillating hydrodynamic lift force acting on the cylinder and is given by Equation (2). 
The amplitude of oscillation of the system depends on the mass $\left(m^{*}\right)$ ratio of the oscillating cylinder given by

$$
m^{*}=\frac{m}{m_{f d}}
$$

where $m_{f d}$ is the mass of fluid displaced by the oscillating mass. The maximum possible response amplitude at any $R e, Y_{\max }$ can be calculated from the empirical relation between non-dimensional amplitude $\left(A_{y}=\frac{Y_{\max }}{D}\right)$ and Re [10] as given by Equation (7).

$$
A_{Y}=-0.4435\left[\log \frac{\alpha}{R e}\right]-1.5
$$

where $\alpha$ is defined as

$$
\alpha=\left(m^{*}+C_{A}\right) \xi
$$

where $C_{A}$ is the added mass coefficient and $\xi$ is the damping ratio.

Maximum amplitude of oscillation occurs when shedding frequency locks on to the natural frequency of the oscillating system $\left(f_{n}\right)$. This condition is known as lock-in. Amplitude of oscillation of a spring mass system can also be obtained from Equation (9)

$$
Y=\frac{F_{L}}{k}\left[\frac{1}{\sqrt{\left(1-\eta^{2}\right)^{2}+(2 \xi \eta)^{2}}}\right]
$$

where $\eta$ is the frequency ratio represented by Equation (10)

$$
\eta=\frac{f_{n}}{f_{v}}
$$

where $f_{v}$ is the vortex shedding frequency. During lock-in $\eta=1$ and Equation (9) simplifies as shown by Equation (11).

$$
Y=\frac{F_{L}}{2 k \xi}
$$

The paper discusses effect of mass and stiffness ratios of the oscillating cylinder on the maximum response amplitude. The structural damping variations are not considered as it is observed to be less significant compared to its inertia and elastic counterparts. Also, mass and stiffness of the system are more tangible parameters from design point of view compared to damping. Moreover, the study focuses on non-dimensionalizing the influencing parameters so that results hold applicable for prototypes and models equally. Many researchers have considered the combined effect of mass and damping through mass damping parameter $m^{*} \xi$. In such analysis also $\xi$ is kept constant and $m^{*}$ is varied independently [17].

\section{Numerical Determination of Hydrodynamic Lift Forces and Motions}

Vortex induced vibration, a two-way fluid structure interaction phenomenon, is complex in nature due to the fact that the cylinder displacement is capable of changing the vortex shedding pattern behind it leading to a variation in the hydrodynamic load acting on the cylinder. Parameters $k^{*}, m^{*}$, and $c$ have significant influence on the oscillation amplitude. A significant amount of research has been carried out to bring clarity on the influence of these parameterson the maximum cylinder response amplitude. Experiments conducted by [18] could capture lock-in phenomenon for a cylinder with a single degree of freedom. It was observed that cylinder oscillation frequency matches with vortex shedding frequency for cylinders having low mass ratios [19]. For mass ratios below critical mass ratio, $m_{c r}^{*}$, the range of $U_{r}$ over which resonance occurs tend to extend towards infinity. The following section of the paper is an effort to understand the effect of mass and stiffness coefficient on the response 
of cylinder numerically in a simpler and economic way, and the results of the numerical study is verified using a field test.

\subsection{Modeling the Flow}

Numerically this problem has been treated as a case of two-way fluid structure interactions (two-way FSI). Modeling and meshing has been performed in ANSYS ICEM CFD (version 12) [20] and solving using ANSYS FLUENT (version 12) [21]. Flow around the cylinder is modeled using the transient, incompressible Navier-Stokes equation based RANS solver with $k-\omega \mathrm{SST}$ as the turbulence model. The RANS solver does the virtual averaging of velocities over an interval of time and hence for a specific interval the velocity vector appears to be constant in a RANS solver. In the present work, an optimized fine grid is used to compensate for this drawback of the solver enabling it to capture the physics of von Kármán street eddies. RANS solver for transient two-dimensional analysis can be explained as follows [21]

$$
\begin{gathered}
\frac{\partial \rho}{\partial t}+\frac{\partial}{\partial x_{i}}\left(\rho u_{i}\right)=0 \\
\frac{\partial}{\partial t}\left(\rho u_{i}\right)+\frac{\partial}{\partial x_{i}}\left(\rho u_{i} u_{j}\right)=-\frac{\partial p}{\partial x_{i}}+\frac{\partial}{\partial x_{j}}\left[\mu\left(\frac{\partial u_{i}}{\partial x_{j}}+\frac{\partial u_{j}}{\partial x_{i}}-\frac{2}{3} \delta_{i j} \frac{\partial u_{l}}{\partial x_{l}}\right)\right]+\frac{\partial}{\partial x_{j}}\left(-\rho \overline{u_{i}^{\prime} u_{j}^{\prime}}\right)
\end{gathered}
$$

where $u_{i}$ and $u_{i}^{\prime}$ are mean and fluctuating velocity components for $i=1,2$, and 3 .

The velocities and other solution variables in the above equation represent the time averaged values. Equation (12) is solved by modeling Reynolds stresses $\rho \overline{u_{i}^{\prime} u_{j}^{\prime}}$, effectively using $k-\omega$ SST as turbulence model [22]. $k-\omega$ SST is capable of accurately predicting the commencement and the intensity of flow separation at fixed boundaries while the standard $k-\varepsilon$ model has proved its efficacy in predicting the wake characteristics accurately. This fact has been established after extensive studies conducted by the authors. $k-\omega S S T$ turbulence model demands a very high near wall grid resolution and hence the maximum element size is fixed to be less than $1 \times 10^{-4}$, which satisfies CFL criterion and near wall $y+$ values.

The above governing equations are discretized using finite difference method. Non iterative time advancement (NITA) scheme with fractional time stepping method (FSM) has been chosen for pressure-velocity coupling of the grid. A Least Squares Cell Based scheme is used for gradient in spatial discretization and a second order upwind scheme as convective scheme.

\section{NITA}

For capturing the physics of the flow with accuracy in the boundary as well as in the wake of the cylinder, the computational grid needs to be extremely fine. Solving a dynamic mesh case with such extreme grid fineness using iterative time advancement scheme demands a considerable number of iterations to be performed using a very small time step size to satisfy the dynamic mesh criteria. This in turn leads to huge computational cost and effort. As an alternative and computationally economic method, in the present work, NITA with FSM has been implemented. NITA scheme assures the same time accuracy by reducing the splitting error, which occurs while solving the discretized Navier-Stokes equation to the same order as the truncation error. Splitting error need not be reduced to zero in NITA scheme, saving a lot of computational effort.

\subsection{Structural Modeling}

An elastically mounted cylinder can be mathematically represented by Equation (4). This equation of motion is solved using six degrees of freedom solver (6DOF), an integral part of the main solver by defining the cylinder as an object with one degree of freedom (1DOF) in transverse direction. A user defined function (UDF) compiled in C programming language has been hooked to the cylinder dynamic boundary conditions. The governing equation for the motion of the center of gravity of 
the cylinder in the transverse direction is solved in the inertial coordinate system. Velocity in the transverse direction is obtained by performing integration on Equation (14).

$$
\ddot{Y}=\frac{1}{m} \sum F
$$

where $\ddot{Y}$, is the translational acceleration in the transverse direction, $\mathrm{m}$ is the mass of the cylinder and F, resultant fluid force acting on the cylinder. Position of the center of gravity of the cylinder (CG) is updated after solving the equation of motion of a spring mass system.

$$
m \ddot{Y}+c \dot{Y}+k Y=F(t)
$$

The inertial force term on the left-hand side of Equation (15) is computed by the 6DOF solver for each time step from Equation (14) and the UDF hooked to the moving cylinder inputs the restoring force term as $6 \mathrm{DOF}$ load acting on the cylinder. Mass of the cylinder is given in the UDF as

$$
\begin{gathered}
m=m_{b}+m_{a} \\
m_{a}=\left(1+C_{A}\right) m_{b}
\end{gathered}
$$

Added mass coefficient $C_{A}$ for the aspect ratio of the present model is found to be equal to 0.7 [13].

\subsection{Mesh Deformation}

Mesh motion to adapt to the movement of the cylinder is achieved by using displacement based smoothing algorithm. The governing equation for mesh motion is represented by Equation (18).

$$
\nabla \cdot(\gamma \nabla \vec{u})=0
$$

where $\vec{u}$ is the velocity of mesh displacement. The boundary conditions for Equation (18) are computed by the $6 \mathrm{DOF}$ solver and the boundary mesh motion diffuses into the interior of the deforming mesh according to the Laplace equation, Equation (18). Diffusion coefficient, $\gamma$ is calculated using boundary distance formulation given by Equation (19).

$$
\gamma=\frac{1}{d^{\tau}}
$$

where $\tau$ is the diffusion parameter and $d$ is the normalized boundary distance. Diffusion parameter is set as unity to avoid excessive deformation of the near cylinder elements.

\subsection{Fluid Structure Interaction}

In this paper, a two-way implicit approach is used to study the effect of $m^{*}$ and $k^{*}$ on the response of cylinder under VIV. Flow equations and structural equations are solved simultaneously in iterations with a time step. A flow chart for the solution procedure is shown in Figure 2.

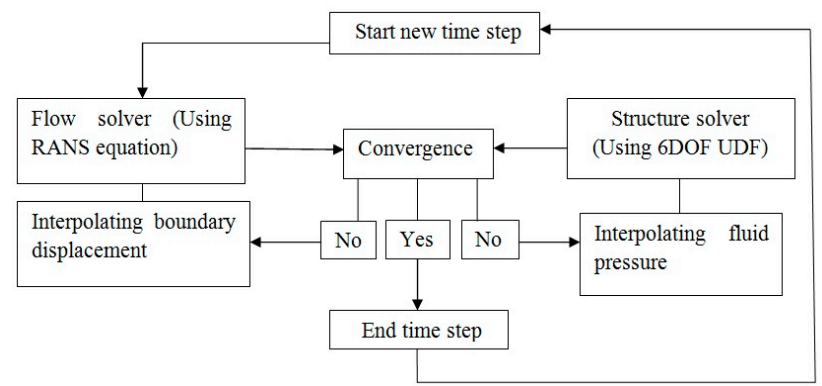

Figure 2. Flow chart of two-way implicit FSI solution procedure. 


\section{Problem Description}

\subsection{Simulation Parameters}

In the present study, geometrically identical cylinders of different masses are considered for VIV of an elastically mounted horizontal cylinder. Based on the present study, a basic model of HVPG as in [5] was fabricated and a field study was carried out on it for verifying the numerical results. The main parameters of the model are summarized in Table 1. The model has an aspect ratio of 13.12 and an outer diameter of $0.0762 \mathrm{~m}$. The structural damping is considered to be zero in the present study. The computational domain for the study is shown in Figure 3a. The representation of the present problem as a spring-mass system is depicted in Figure $3 \mathrm{~b}$. The mesh fineness in indicated in Figure $3 \mathrm{c}$ and the grid used for the present computation is represented by Figure $3 \mathrm{~d}$.

Table 1. Properties of the elastically mounted cylinder model.

\begin{tabular}{ccc}
\hline Properties & Values & Units \\
\hline Diameter of the cylinder $(D)$ & 0.0762 & $\mathrm{~m}$ \\
Aspect ratio of the cylinder $(L / D)$ & 13.12 & - \\
Flow velocity $(V)$ & 0.5 & $\mathrm{~m} / \mathrm{s}$ \\
Reynolds Number of flow $(\mathrm{Re})$ & $3.8 \times 104$ & - \\
Mass ratio $\left(m^{*}\right)$ & 0.66 & - \\
\hline
\end{tabular}

A simple representation of the two-dimensional computational domain for the cylinder is displayed in Figure 3a. The flow direction is parallel to the global $x$-axis and the flow velocity is set to be $0.5 \mathrm{~m} / \mathrm{s}$. Domain size is fixed based on previously published analysis and allowance is given to accommodate the vertical motion of cylinder boundary [23]. Simulations are performed for three different values of mass ratio, $m^{*}=0.66,1.32$, and 1.98 which correspond to moderate mass ratios above $m_{c r}^{*}$, for which maximum response in confined to the range of $U_{r}=4-12$. For fixed mass, influence of stiffness ratio $k^{*}$ is studied by varying the reduced velocity value which is defined as

$$
\begin{gathered}
U_{r}=\frac{V}{f_{n} D} \\
f_{n}=\frac{1}{2 \pi} \sqrt{\frac{k}{m}}
\end{gathered}
$$

Each case has been analyzed over a range of reduced velocity, $U_{r}=4-12$, over which the cylinder is predicted to have maximum amplitude of oscillation [24]. $U_{r}=5$ has also been analyzed since the case corresponds to $\eta=1$ where one might expect resonance. The incoming flow velocity is fixed as $0.5 \mathrm{~m} / \mathrm{s}$ to maintain the flow regime uniform at $R e=3.8 \times 10^{4}$. The mass ratio, stiffness ratio, and other parameters for each case are summarized in Table 2. Stiffness coefficient of the cylinder has been non-dimensionalized to generalize the applicability of the analysis. Stiffness ratio is defined as

$$
k^{*}=\frac{k}{m g / L}
$$

Table 2. Reduced velocity, stiffness ratio, and frequency ratio at $R e=3.8 \times 10^{4}$, for $m^{*}=0.66,1.32$, and 1.98 .

\begin{tabular}{ccc}
\hline$U_{r}$ & $k^{*}$ & $\eta$ \\
\hline 4 & 11.17 & 1.3 \\
5 & 6.9 & 1.0 \\
6 & 4.81 & 0.84 \\
8 & 2.7 & 0.63 \\
10 & 1.73 & 0.51 \\
12 & 1.21 & 0.42 \\
\hline
\end{tabular}


SYMMETRY

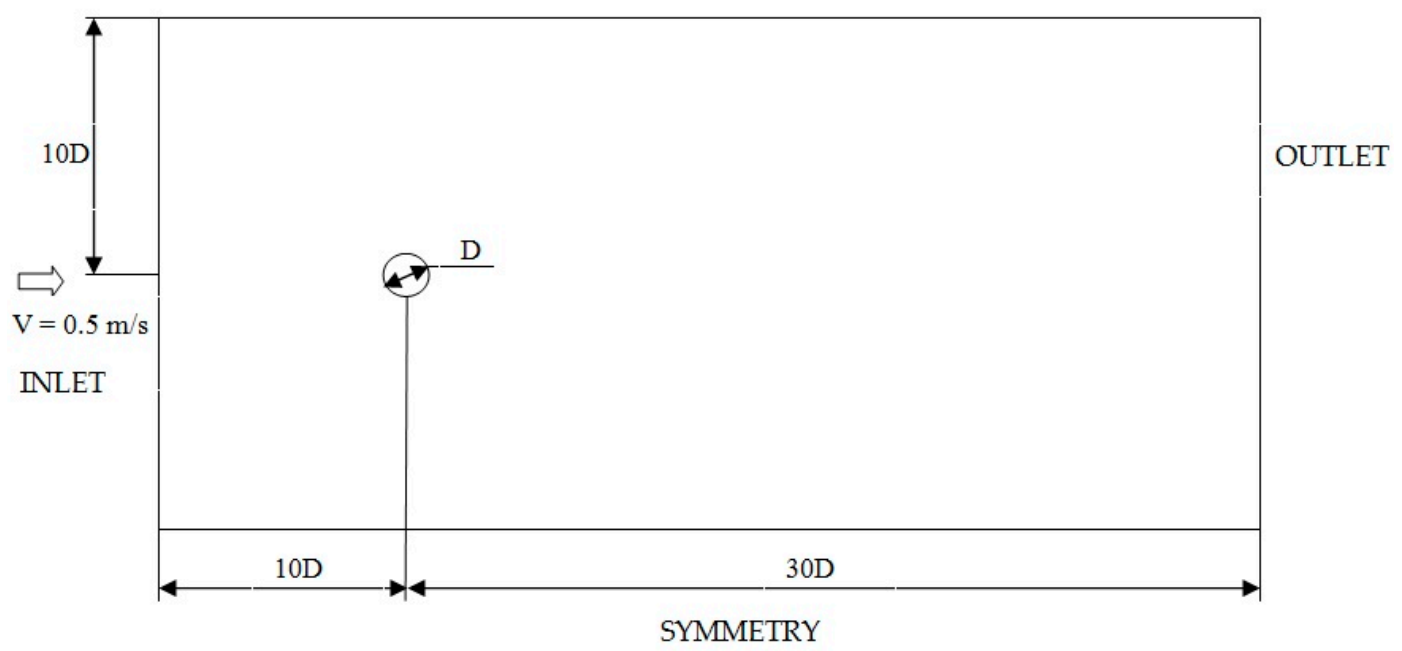

(a)

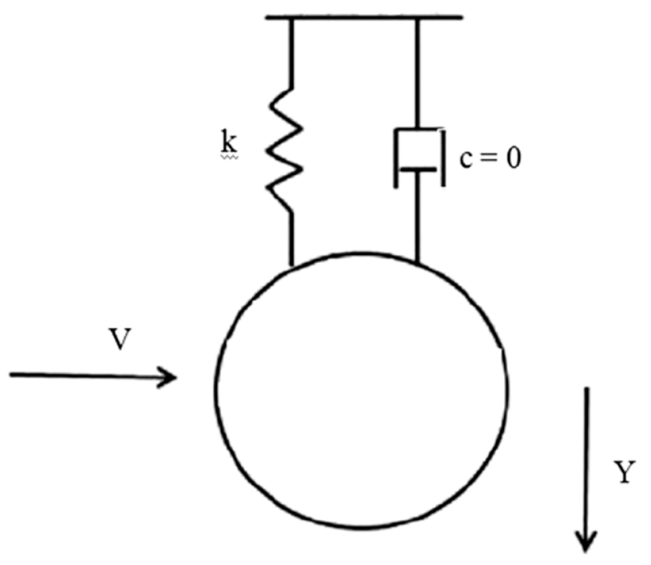

(b)

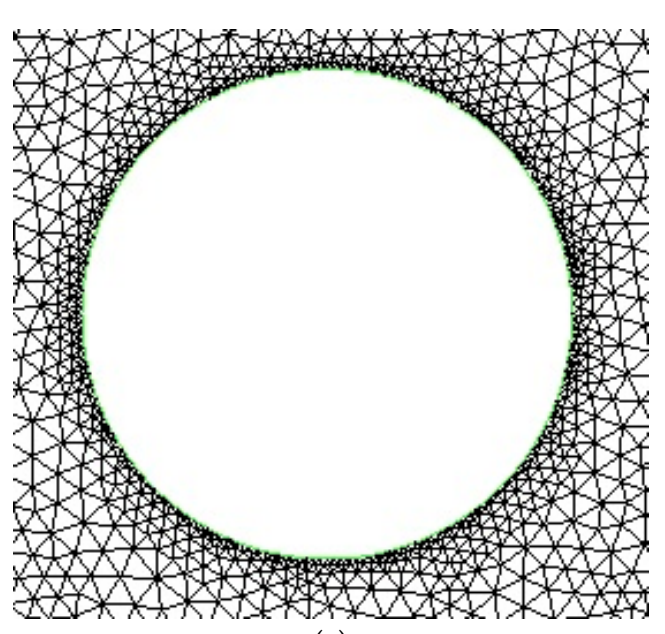

(c)

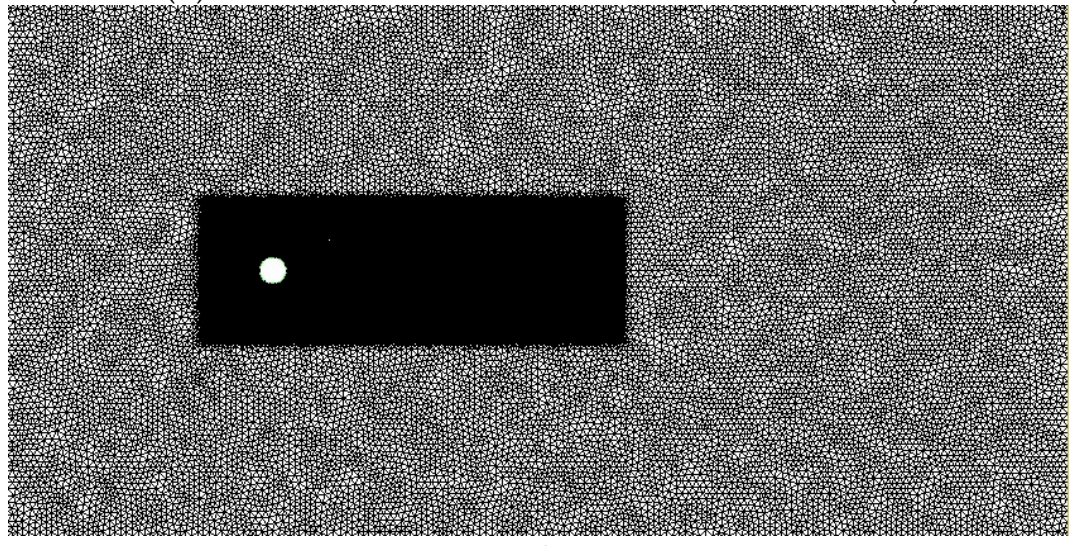

(d)

Figure 3. (a) Computational domain; (b) representation of elastically mounted cylinder model; (c) mesh around the cylinder; (d) computational mesh.

\subsection{Fluid Domain and Boundary Conditions}

Figure 3a shows the computational domain for the CFD simulation of VIV of an elastically mounted horizontal cylinder. The origin of the Cartesian coordinate system is located at the center of the cylinder. The length of the domain is 40D with the cylinder located at $10 \mathrm{D}$ away from the inlet boundary. The cross-flow width of the domain in 20D with the center of the cylinder at the 
middle. Detailed views of the mesh around the cylinder along with the computational domain after meshing have been shown in Figure $3 c$,d respectively. There are 307 nodes around the circumference of the cylinder and the minimum element size near the rigid wall boundary has been computed from boundary layer theory to be $0.0001 D$. The non-dimensional element size represented as $y+$, next to the cylinder surface is found to be less than unity. For the cylinder wall, a no slip boundary condition has been applied assuming the surface to be smooth. Inlet boundary has been treated has velocity-inlet with inflow velocity, $V=0.5 \mathrm{~m} / \mathrm{s}$. Outlet boundary has been treated as pressure outlet, the gradients of fluid velocity are set to zero and the pressure with zero reference pressure. On the two transverse boundaries a symmetry boundary condition has been applied.

\subsection{Mesh Independence Study}

An unstructured 2D mesh has been used in the present CFD simulation to facilitate computationally economic platform for dynamic mesh simulation. The three dimensionality of the wake reduces as a result of the motion of the cylinder [16]. Hence it is possible to get a reasonably accurate result from a $2 \mathrm{D}$ analysis saving much computational cost and effort. The meshing strategy is that finer mesh is used in the vicinity of the moving cylinder with extra fine meshing in the boundary layer. Boundary layer thickness and the near wall element size have been calculated from boundary layer theory. The thickness of laminar sub-layer is obtained from Equation (23) [25]

$$
\delta^{\prime}=\frac{11.6 \vartheta}{V^{*}}
$$

where $V^{*}$ is the frictional velocity given by

$$
V^{*}=\sqrt{\frac{\tau_{0}}{\rho}}
$$

and $\tau_{0}$, the wall shear stress is obtained as

$$
\tau_{0}=\frac{0.664}{\sqrt{R e_{D}}} \cdot \frac{\rho V^{2}}{2}
$$

Increased mesh density has been adopted in the near cylinder and its wake in order to capture the physics of vortex shedding accurately. For ensuring that the results are independent of the grid size, a mesh independence study has been carried out. Three different grids have been used to simulate a specific case $m^{*}=2.45$ and $U_{r}=8$ and has been verified using results of experiments conducted at a towing tank facility in the Department of Ocean Engineering, Indian Institute of Technology Madras, India [10]. The details of the mesh independency study are given in Table 3. The last three grids give almost similar results and experiments, and [10] shows a $4.9 \%$ deviation from the present results. This can be assumed to be due to not accounting for damping in the present study. It can be concluded that the variation in the numerical results given by Meshes II, III, and IV are in the acceptable range and considering the computational economy Grid II with 49,995 nodes has been chosen for further analysis.

Table 3. Mesh independency study results

\begin{tabular}{cccccc}
\hline & $\boldsymbol{R} \boldsymbol{e}$ & $\boldsymbol{m}^{*}$ & $\boldsymbol{U}_{\boldsymbol{r}}$ & Nodes & $\boldsymbol{Y}_{\max } / \boldsymbol{D}$ \\
\hline Grid I & & & & 35,487 & 1.241 \\
Grid II & \multirow{3}{*}{$3.8 \times 104$} & 2.45 & 8 & 49,995 & 1.220 \\
Grid III & & & & 70,857 & 1.219 \\
Grid IV & & & & 98,475 & 1.219 \\
\hline Narendran et al. (2015) & $0.3-2.4 \times 105$ & 2.45 & 8 & - & 1.160 \\
\hline
\end{tabular}


While due steps like mesh independence studies have been carried out in this investigation, we acknowledge that the use of eddy-viscosity based turbulence models introduces a small amount of discrepancy in the final results. The errors of the $k-\omega$ SST model in flow separation and wakes has been documented comprehensively in prior studies [26,27].

\section{Results and Discussion}

Numerical simulations have been carried out for three different mass ratios over reduced velocities ranging from 4 to 12 . The cylinder is modeled to be having only single degree of freedom (SDOF) in the transverse or CF direction. Influence of $m^{*}$ and $k^{*}$ on both hydrodynamic force coefficient in the CF direction and the response of the cylinder have been studied in detail. The CF response results can be verified with experimental $[17,28]$. Time history of coefficient of lift, $C_{L}$ and non-dimensional CF response $Y / D$ over the range of $U_{r}$ for $m^{*}=0.66,1.32$, and 1.98 are displayed in Figures $4-6$ respectively.

\subsection{Case I}

Under Case I, the response of cylinder is studied for $m^{*}=0.66$. The mass of the cylinder is taken as $3 \mathrm{~kg}$ and added mass coefficient $C_{A}=0.7 . U_{r}$ is varied in the analysis by varying the coefficient of stiffness $k$ and in turn the natural frequency $f_{n}$ of the oscillating system. Details of the simulation parameters have been given in Table 2. For a stationary cylinder, it was observed from numerous experimental and numerical works [29-31] that $C_{L}$ oscillates in a symmetrical fashion about zero due to vortex shedding [13]. In the present study for lower values of $U_{r}, C_{L}$ is not oscillating about zero symmetrically. However, the response of the cylinder is observed to be symmetrical for all values of $U_{r}$ at $m^{*}=0.66$. At $U_{r}=8, C_{L}$ becomes almost symmetrical with an effective lift coefficient 0.18 . Beat phenomenon is captured in the response of the cylinder at $U_{r}=8$. Results for different cases are presented in Table 4. Maximum lift force is observed at $U_{r}=4$ with $C_{L}=1.12$ and maximum response at $U_{r}=8$ with $Y / D=1.26$. With increase in $U_{r}$ response amplitude of the cylinder decreases beyond $U_{r}=8$. Time histories of $C_{L}$ and $Y / D$ for $m^{*}=0.66$ is presented in Figure 4.

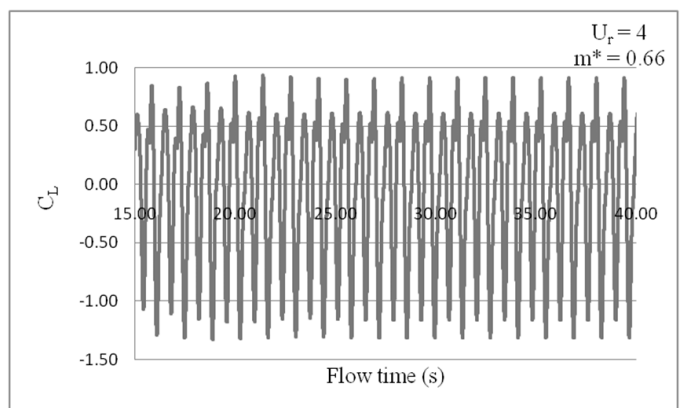

(a)

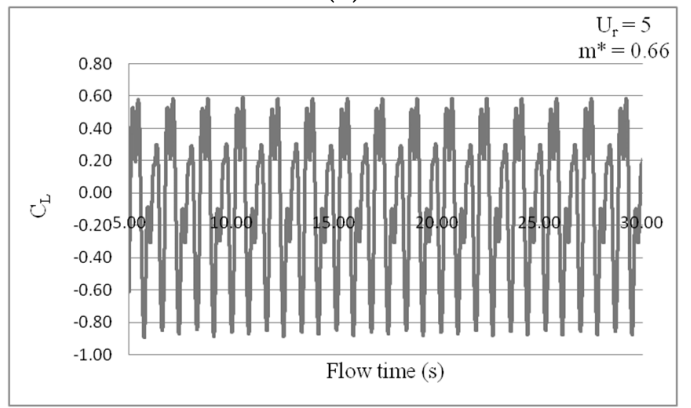

(b)

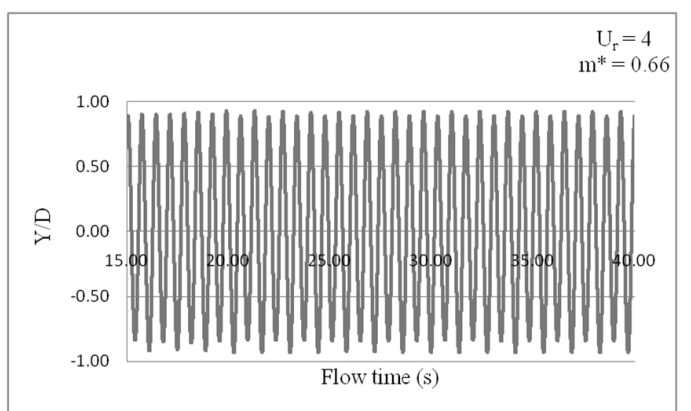

(g)

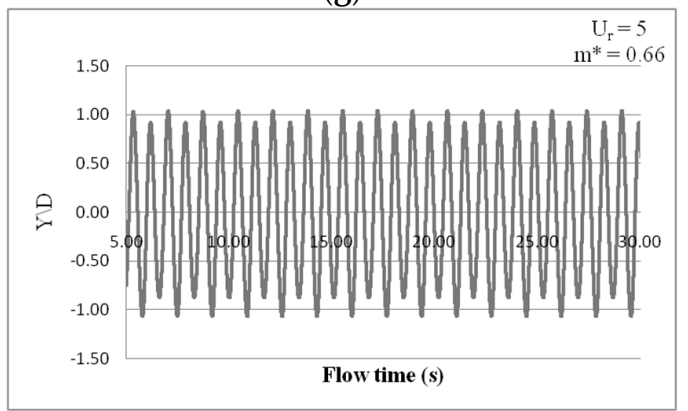

(h)

Figure 4. Cont. 


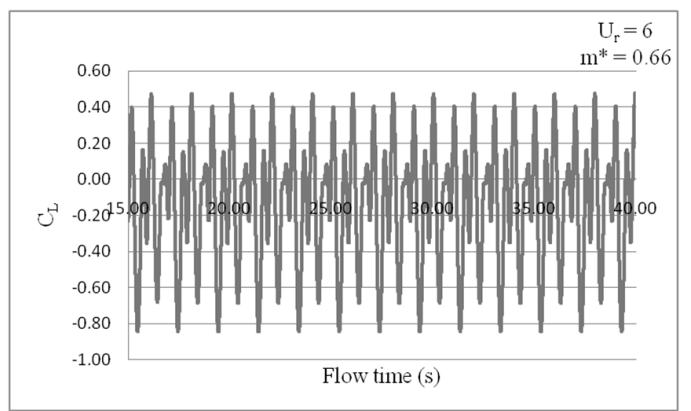

(c)

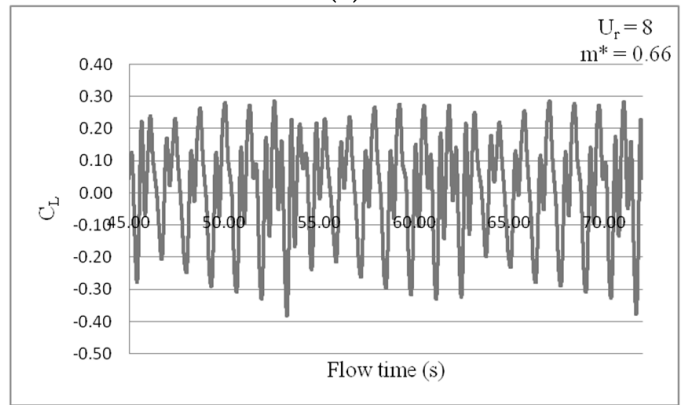

(d)

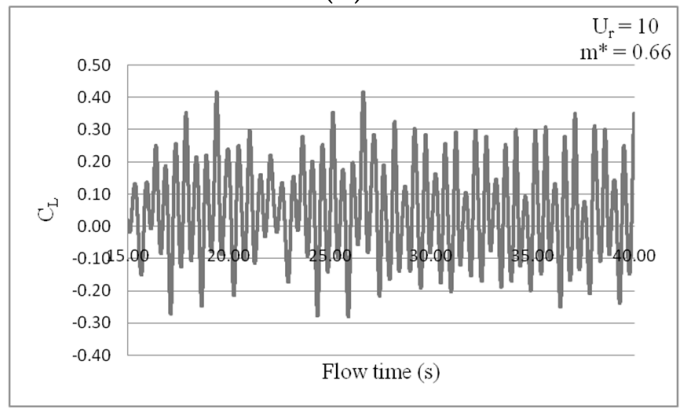

(e)

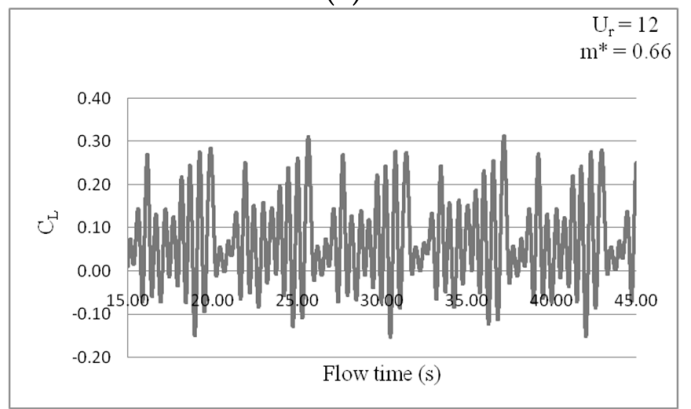

(f)

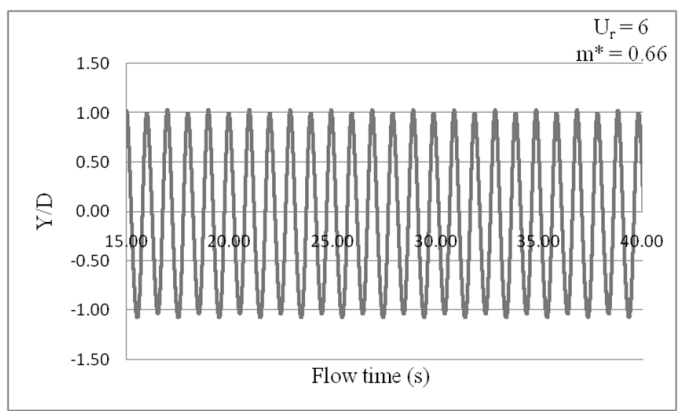

(i)

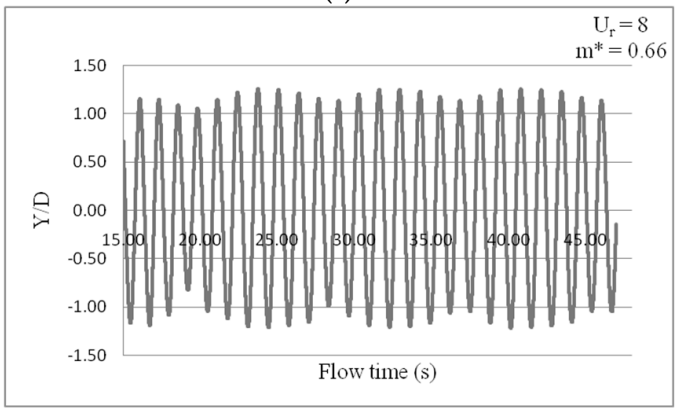

(j)

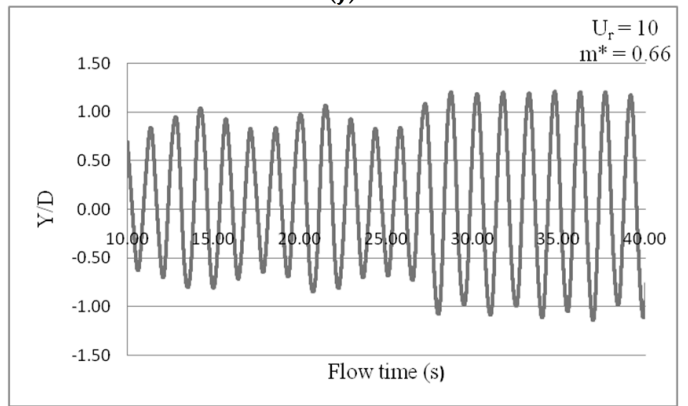

(k)

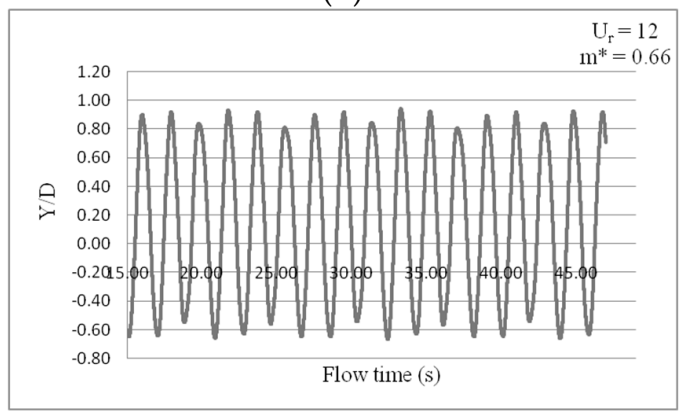

(l)

Figure 4. Time histories of hydrodynamic and structural response parameters at $m^{*}=0.66$ : (a) $C_{L}$ for $U_{r}=4 ;(\mathbf{b}) C_{L}$ for $U_{r}=5 ;(\mathbf{c}) C_{L}$ for $U_{r}=6$; (d) $C_{L}$ for $U_{r}=8$; (e) $C_{L}$ for $U_{r}=10 ;(\mathbf{f}) C_{L}$ for $U_{r}=12 ;(\mathbf{g}) Y / D$ for $U_{r}=4$; (h) $Y / D$ for $U_{r}=5$; (i) $Y / D$ for $U_{r}=6$; (j) $Y / D$ for $U_{r}=8$; (k) $Y / D$ for $U_{r}=10$; (l) $Y / D$ for $U_{r}=12$.

\subsection{Case II}

Under Case II, the response of cylinder is studied for $m^{*}=1.32$. Mass of the cylinder is taken as $6 \mathrm{~kg}$ and added mass coefficient $C_{A}=0.7$. Time histories of $C_{L}$ and $Y / D$ for $m^{*}=1.32$ is presented in Figure 5. At $m^{*}=1.32$, the cylinder exhibits similar response characteristics as in the previous case with maximum response at $U_{r}=8$ with $Y / D=1.17$. As the mass ratio increases, a slight decrease in the cross flow response amplitude is observed. Unlike the previous case, beat phenomenon is observed at $U_{r}=10$. 


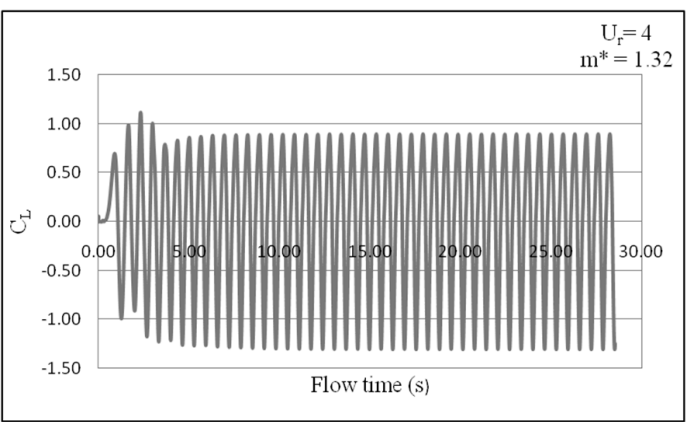

(a)

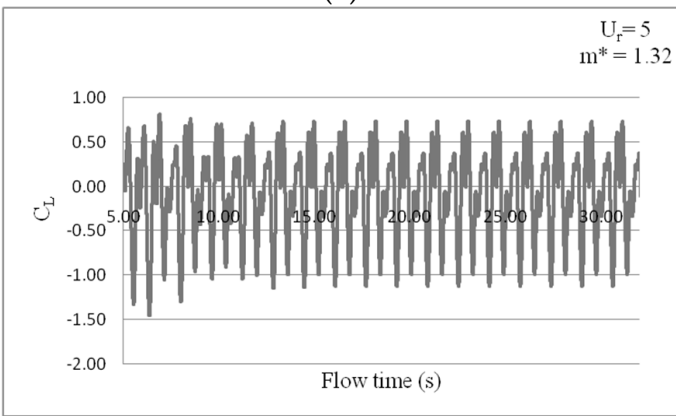

(b)

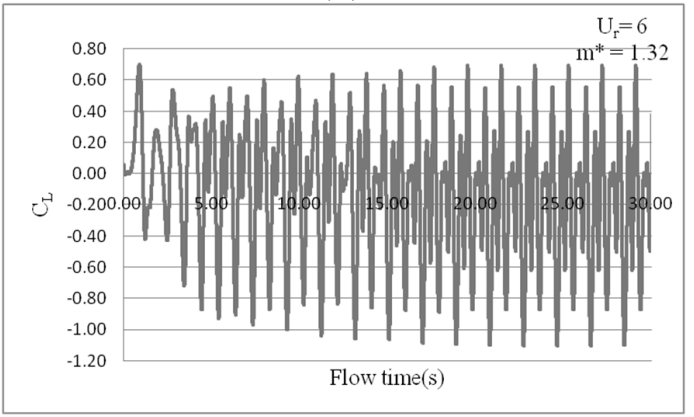

(c)

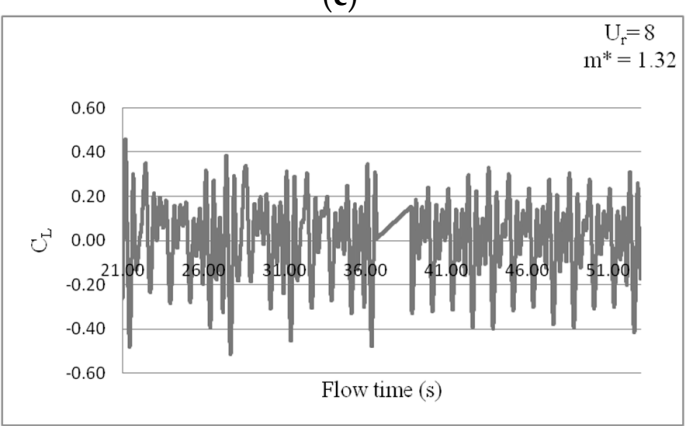

(d)

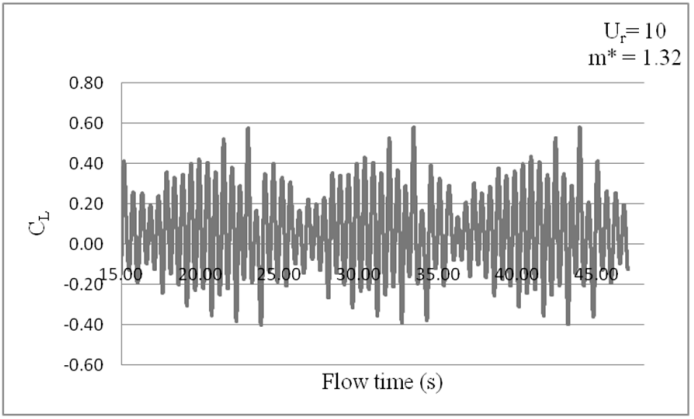

(e)

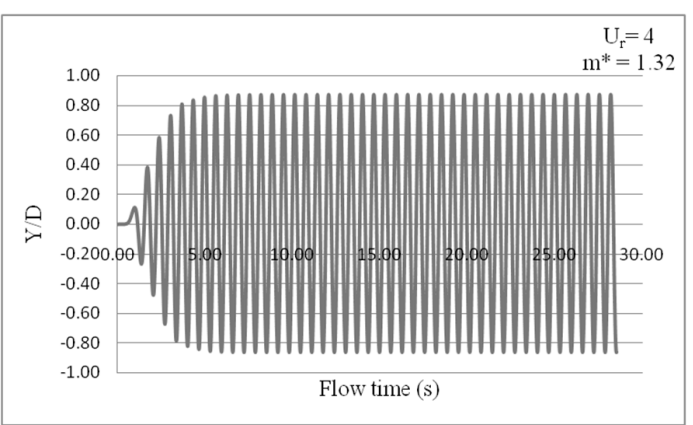

(g)

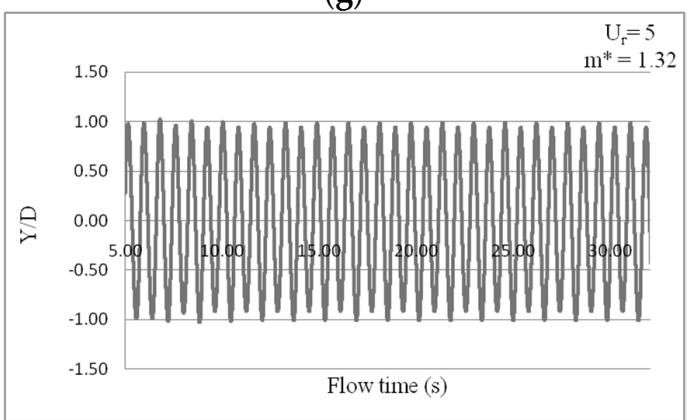

(h)

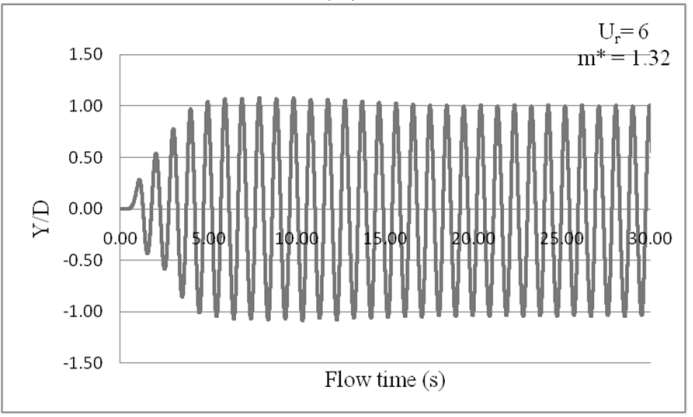

(i)

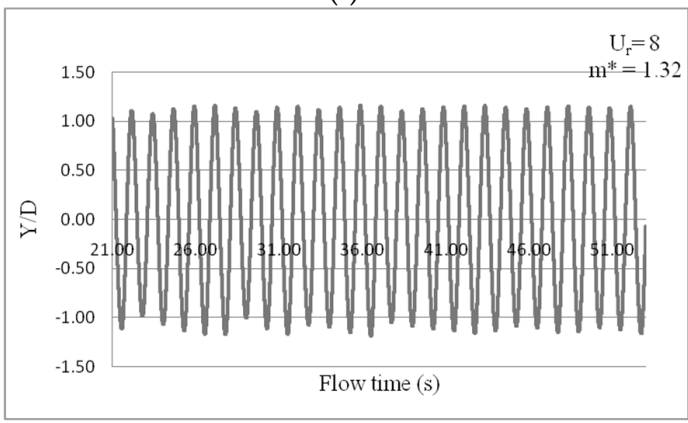

(j)

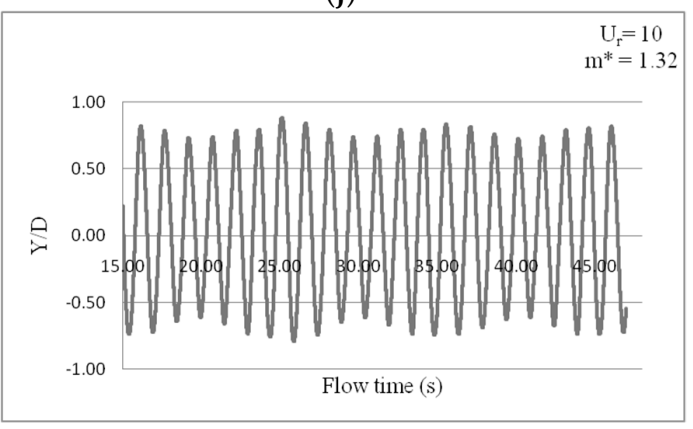

(k)

Figure 5. Cont. 


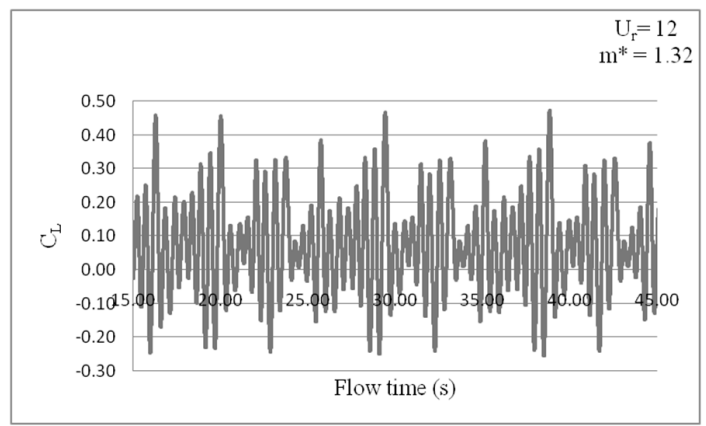

(f)

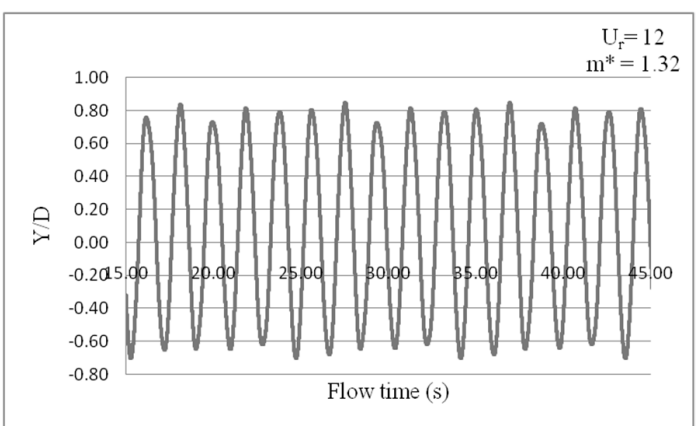

(1)

Figure 5. Time histories of hydrodynamic and structural response parameters at $m^{*}=1.32$ : (a) $C_{L}$ for $U_{r}=4 ;(\mathbf{b}) C_{L}$ for $U_{r}=5 ;$ (c) $C_{L}$ for $U_{r}=6$; (d) $C_{L}$ for $U_{r}=8 ;(\mathbf{e}) C_{L}$ for $U_{r}=10 ;(\mathbf{f}) C_{L}$ for $U_{r}=12 ;(\mathbf{g}) Y / D$ for $U_{r}=4$; (h) $Y / D$ for $U_{r}=5$; (i) $Y / D$ for $U_{r}=6$; (j) $Y / D$ for $U_{r}=8$; (k) $Y / D$ for $U_{r}=10$; (l) $Y / D$ for $U_{r}=12$.

\subsection{Case III}

Under Case III, the response of cylinder is studied for $m^{*}=1.98$. The mass of the cylinder is taken as $9 \mathrm{~kg}$ and added mass coefficient $C_{A}=0.7$. Time histories of $C_{L}$ and $Y / D$ for $m^{*}=1.98$ is presented in Figure 6. Maximum amplitude is observed at $U_{r}=8$ with $Y / D=1.13$ which is slightly less than the previous two cases. Here also prominent beat is observed at $U_{r}=10$.

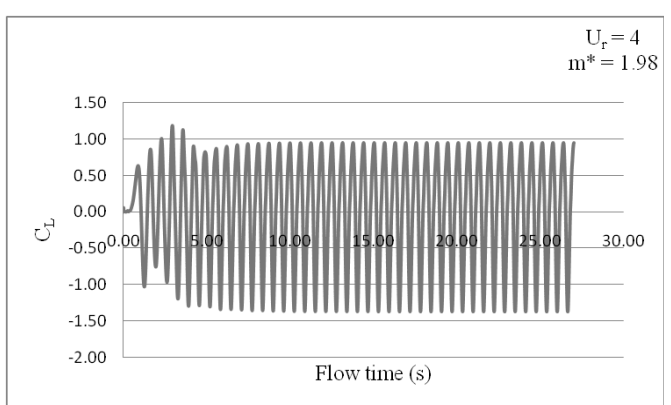

(a)

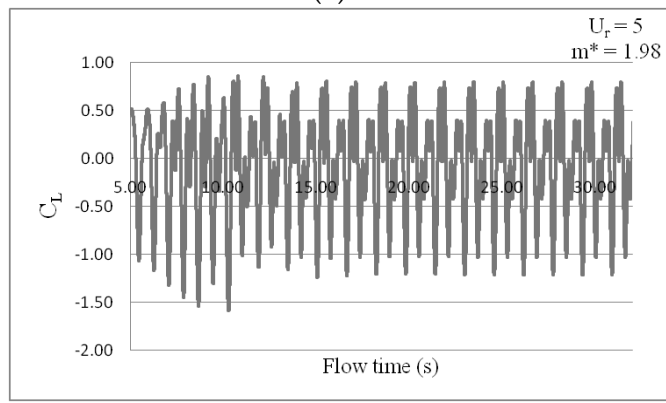

(b)

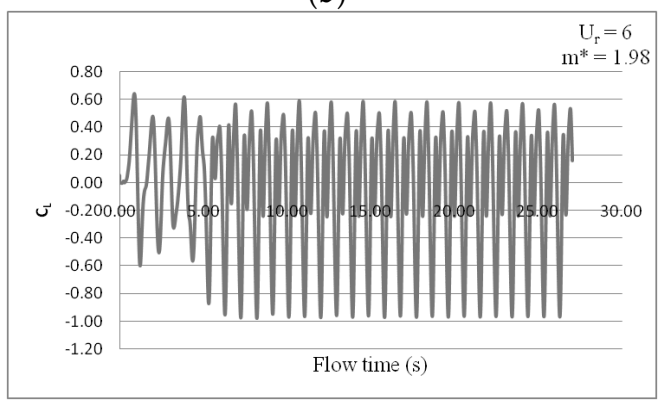

(c)

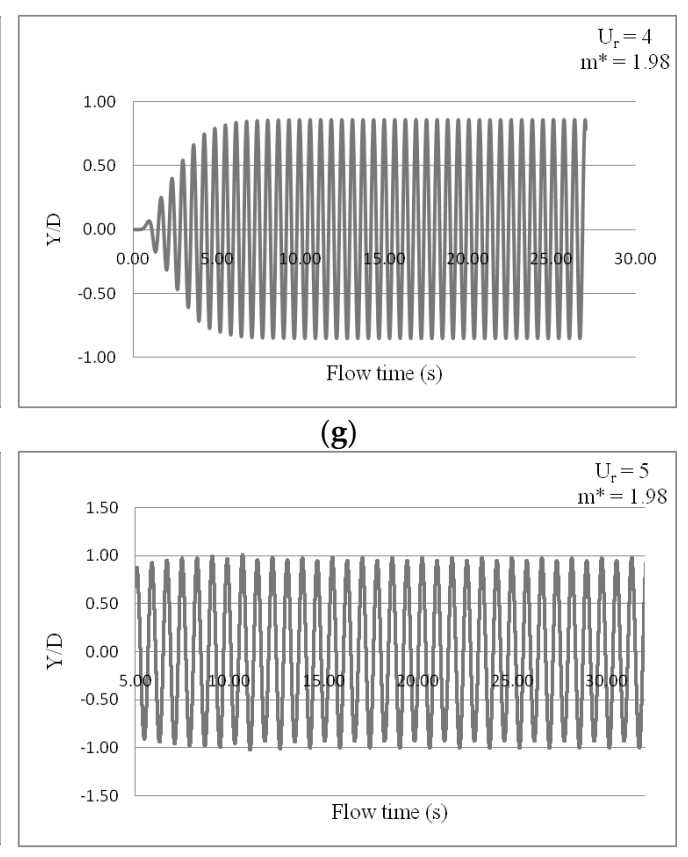

(h)

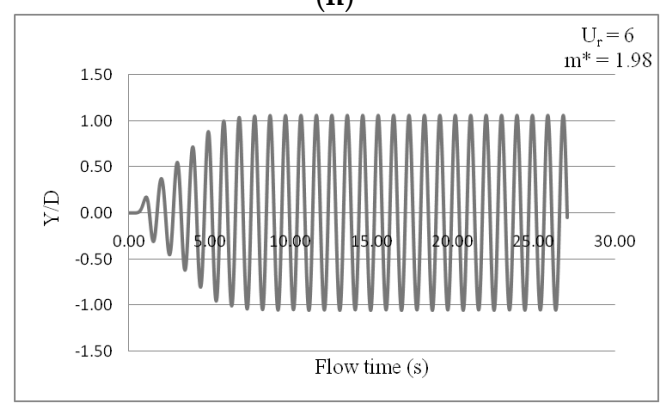

(i)

Figure 6. Cont. 


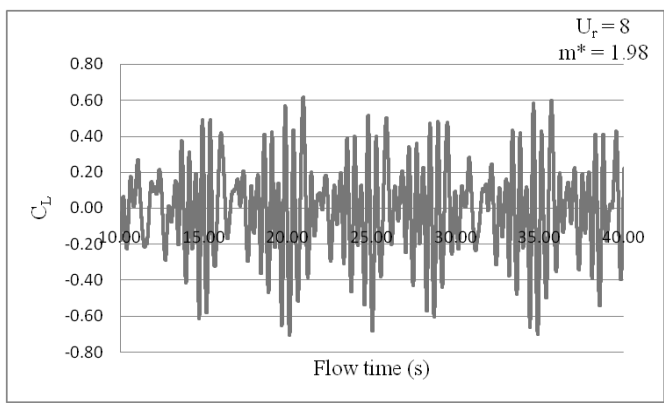

(d)

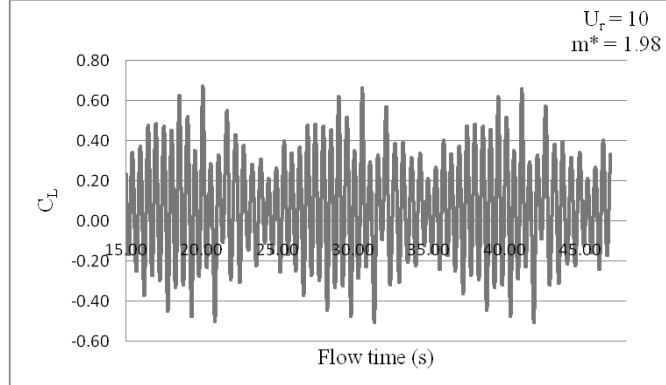

(e)

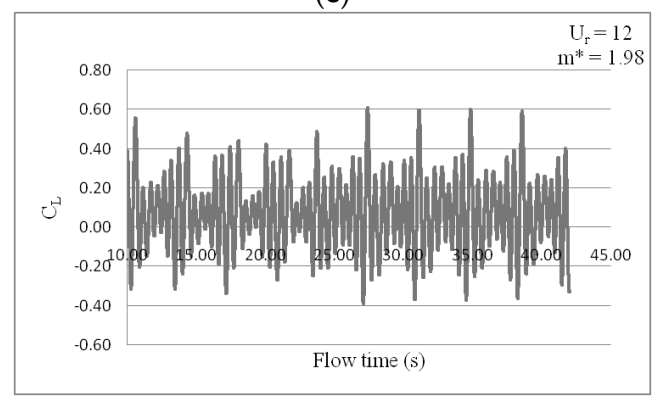

(f)

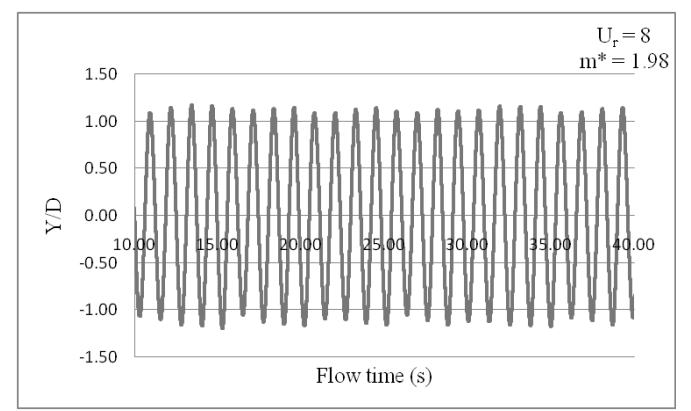

$(\mathbf{j})$

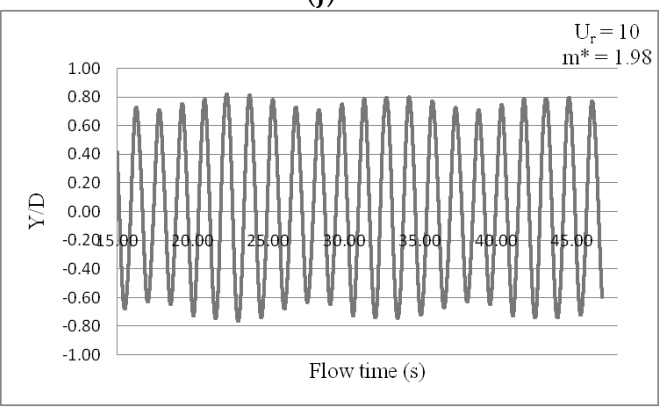

(k)

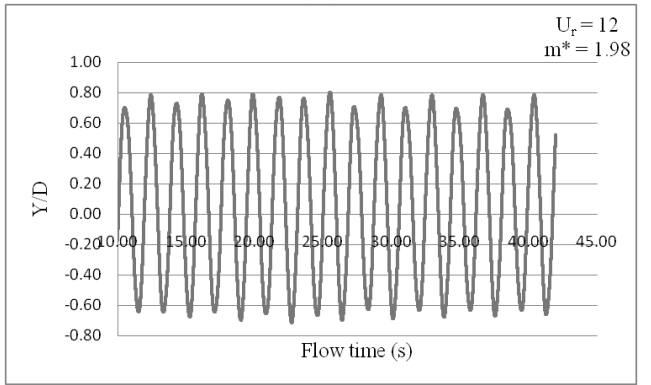

(1)

Figure 6. Time histories of hydrodynamic and structural response parameters at $m^{*}=1.98$ : (a) $C_{L}$ for $U_{r}=4 ;(\mathbf{b}) C_{L}$ for $U_{r}=5 ;$ (c) $C_{L}$ for $U_{r}=6$; (d) $C_{L}$ for $U_{r}=8$; (e) $C_{L}$ for $U_{r}=10 ;(\mathbf{f}) C_{L}$ for $U_{r}=12$; (g) $Y / D$ for $U_{r}=4$; (h) $Y / D$ for $U_{r}=5$; (i) $Y / D$ for $U_{r}=6$; (j) $Y / D$ for $U_{r}=8$; (k) $Y / D$ for $U_{r}=10$; (l) $Y / D$ for $U_{r}=12$.

\subsection{Shedding Characteristics}

Numerical simulations for each case show that the shedding pattern and the characteristic variation of $C_{L}$ strongly depend on the natural frequency of the oscillating mass. A more detailed history of $C_{L}$ and $Y / D$ for $m^{*}=0.66,1.32$, and 1.98 are presented in Figures 7-9 respectively.

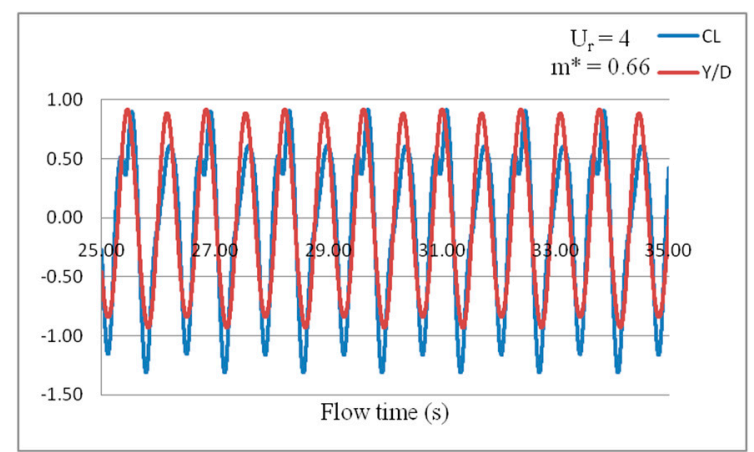

(a)

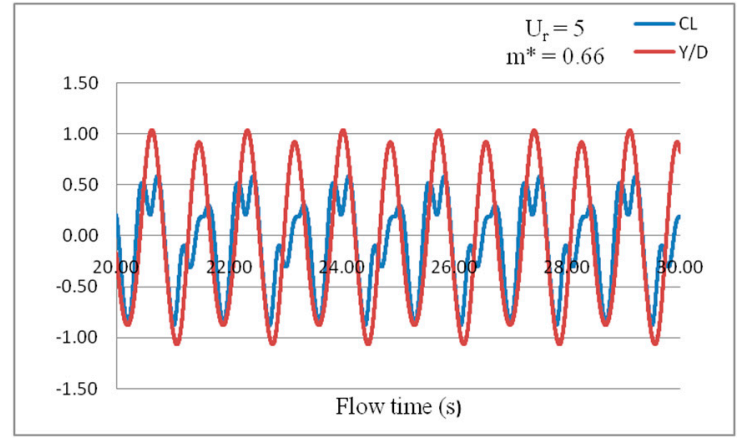

(b)

Figure 7. Cont. 


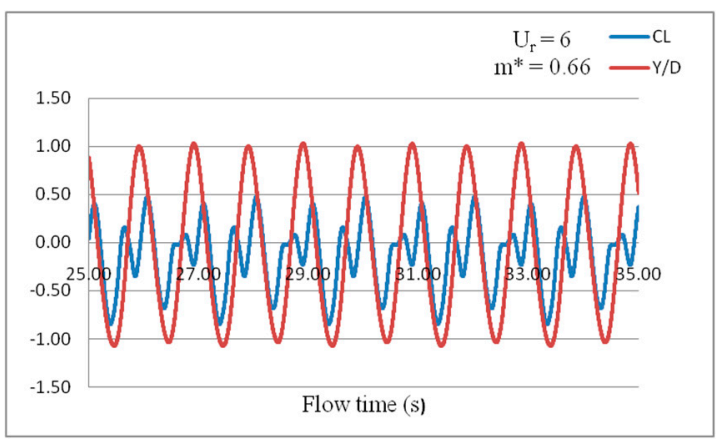

(c)

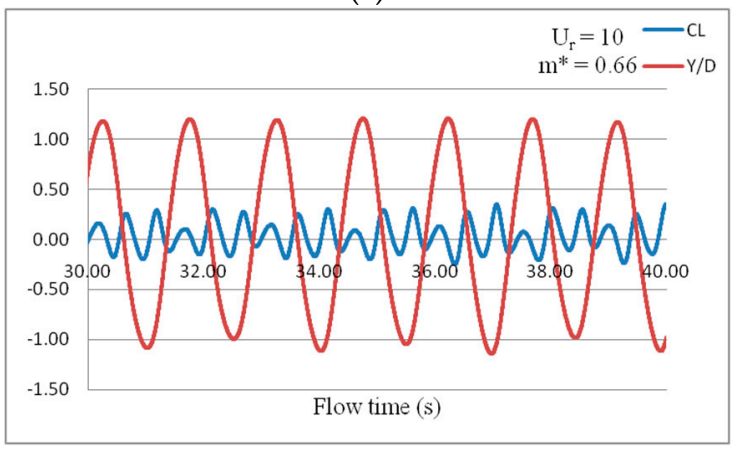

(e)

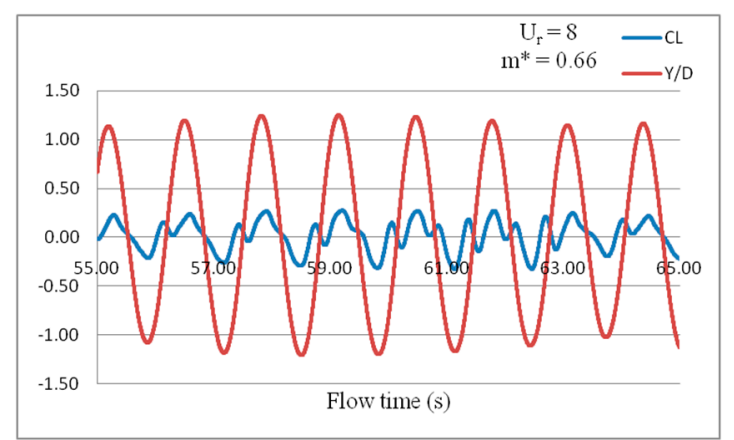

(d)

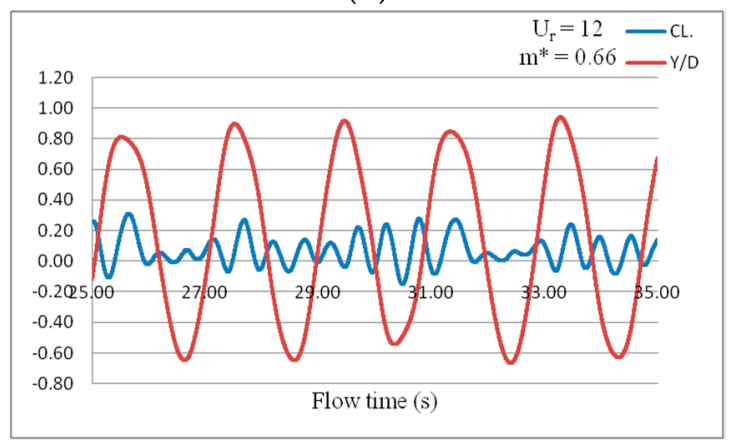

(f)

Figure 7. Variation of $C_{L}$ with $Y / D$ for $m^{*}=0.66$ (a) $U_{r}=4$; (b) $U_{r}=5$; (c) $U_{r}=6$; (d) $U_{r}=8$; (e) $U_{r}=10$; (f) $U_{r}=12$.

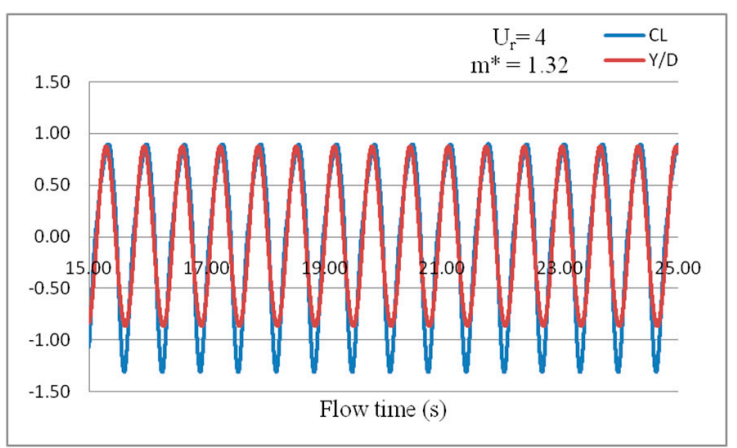

(a)

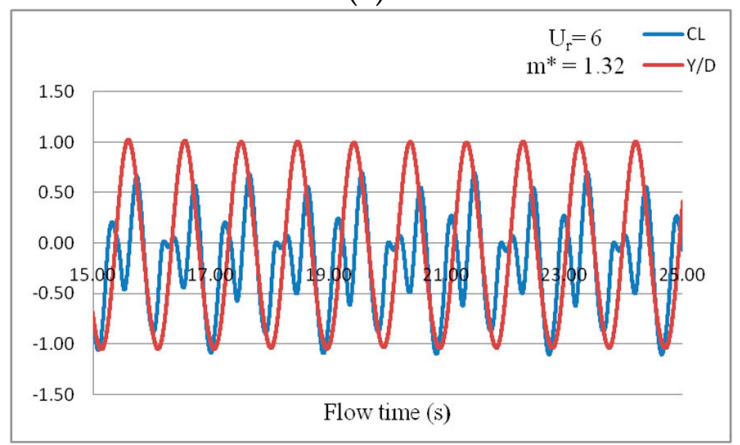

(c)

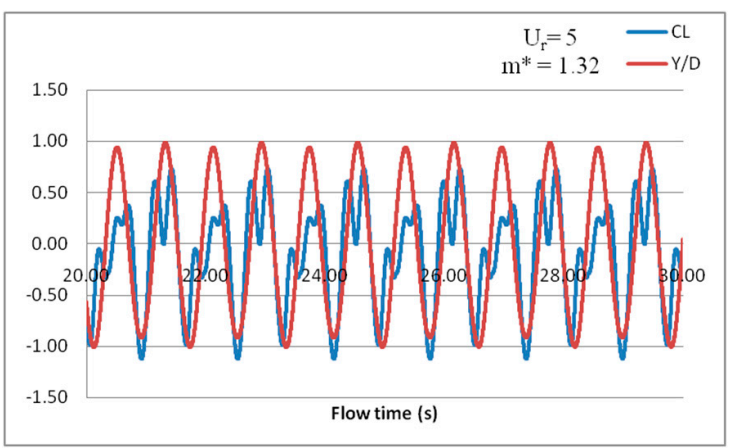

(b)

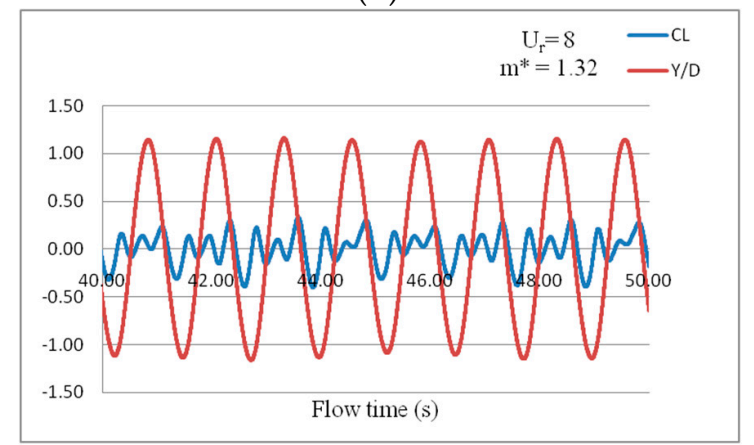

(d)

Figure 8. Cont. 


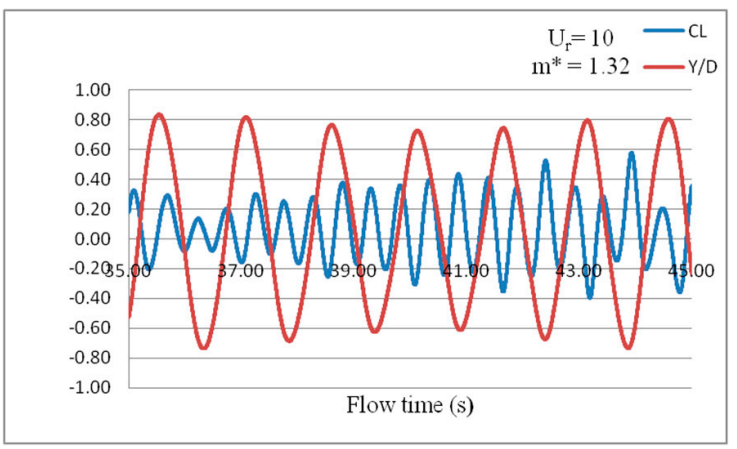

(e)

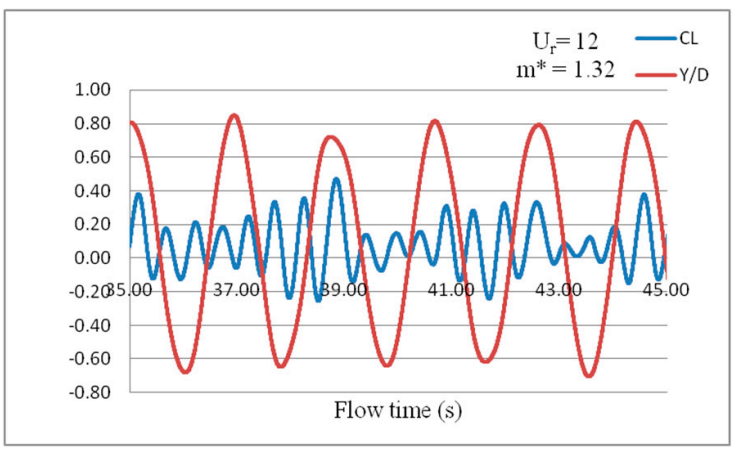

(f)

Figure 8. Variation of $C_{L}$ with $Y / D$ for $m^{*}=1.32$ : (a) $U_{r}=4$; (b) $U_{r}=5$; (c) $U_{r}=6$; (d) $U_{r}=8$; (e) $U_{r}=10$; (f) $U_{r}=12$.

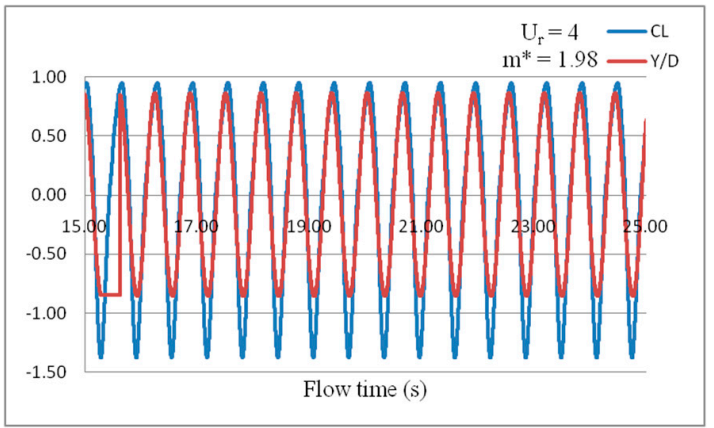

(a)

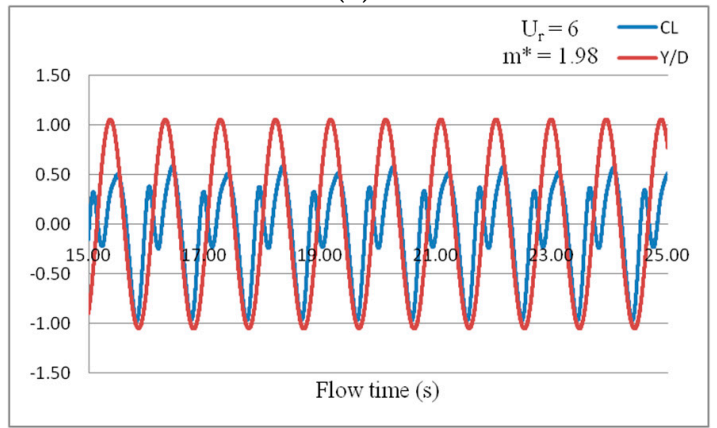

(c)

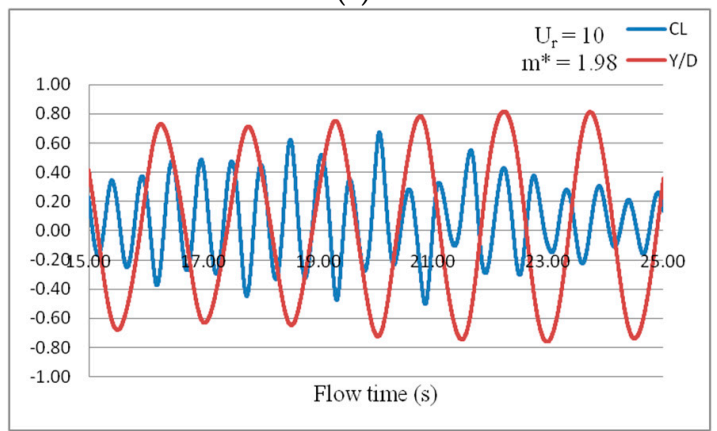

(e)

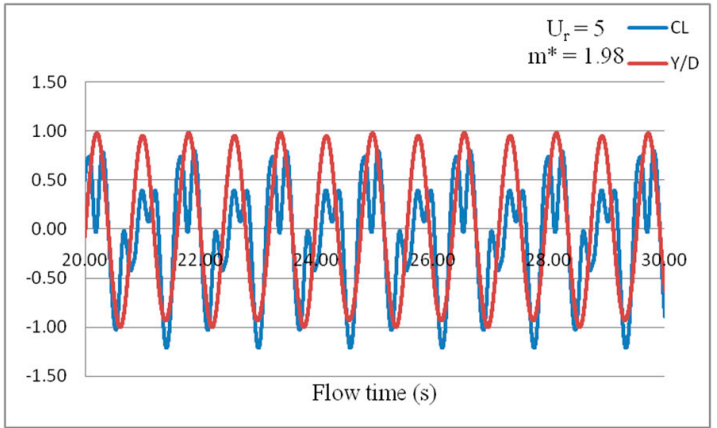

(b)

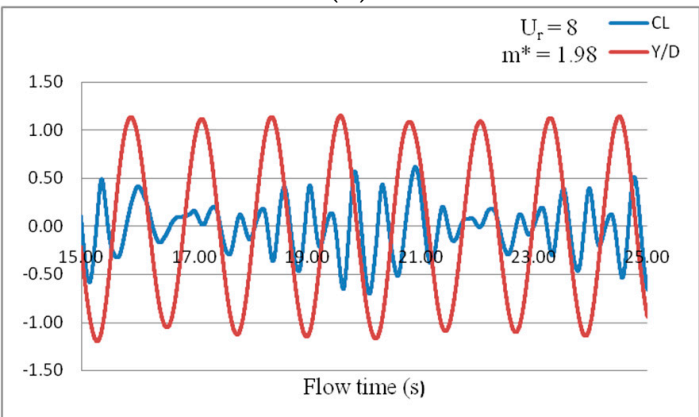

(d)

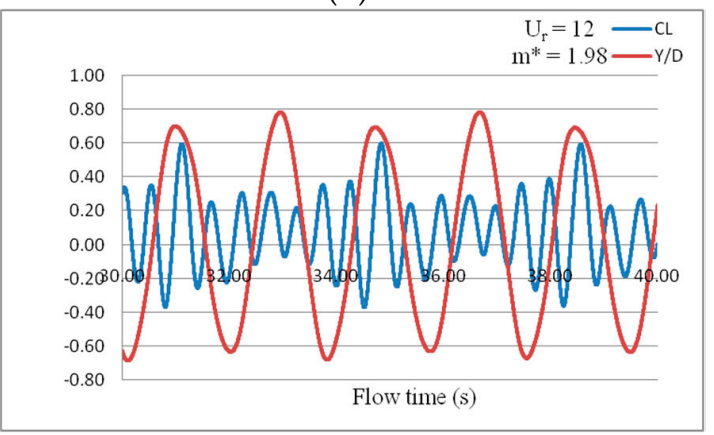

(f)

Figure 9. Variation of $C_{L}$ with $Y / D$ for $m^{*}=1.98$ : (a) $U_{r}=4$; (b) $U_{r}=5$; (c) $U_{r}=6$; (d) $U_{r}=8$; (e) $U_{r}=10$; (f) $U_{r}=12$.

From a detailed analysis of amplitude of variation of lift coefficient, it is observed that the characteristics of hydrodynamic load causing the cylinder to oscillate varies with $U_{r}$ and in turn with frequency ratio, $\eta$. For all the three cases, a similarity can be observed in the shedding characteristics which are reflected in the pattern of lift coefficient variation. In each case, a single oscillation of the 
cylinder is actuated by a single oscillation of lift force when $U_{r}=4$. For $U_{r}=6$, double oscillation of lift coefficient is observed for each oscillation of $Y / D$. The first oscillation is of relatively low amplitude and the second one of larger amplitude. $U_{r}=8$ and 10 which is more prone to beating phenomenon displays three separate $C_{L}$ oscillations for each oscillation of the cylinder. $U_{r}=12$ is observed to have an additional half oscillation. For all mass ratios, a $2 \mathrm{P}$ mode of vortex shedding is observed in the range of $U_{r}=4-12$ which corresponds to synchronization.

A single beat sustains for a time period of 10.53 and 10.42 seconds for $m^{*}=1.98$ and 1.32, respectively. Differently for $m^{*}=0.66$, single beat sustains at $U_{r}=8$ for $7.84 \mathrm{~s}$ which is significantly lesser than the other two cases, showing the highest value of cross flow response amplitude. Also, at the lowest mass ratio, $m^{*}=0.66$, it is observed from the response amplitude history that the cylinder initially shows a tendency to beat at $U_{r}=10$ with a significantly larger response amplitude with $Y / D=1.08$ compared to 0.82 and 0.80 for $m^{*}=1.32$ and 1.98 respectively at same $U_{r}$. Further study on the influencing parameters led to a trend showing a relationship between $m^{*}$ and $U_{r}$, through which they have proved that the range of reduced velocity over which cylinder under VIV shows maximum cross flow response widens as $m^{*}$ decreases [10]. The relationship put forward by [14] is shown in Figure 11. Results of the present study also show that $Y / D$ increases with decreasing mass ratio and the range of $U_{r}$ over which the maximum amplitude of response to be expected widens.

The effect of $U_{r}$ on various simulations parameters is illustrated in Figure 10. A comparison of various hydrodynamic and structural parameters at different $\mathrm{k}^{*}$ values for each case is given in Figure 11a,b. Present simulation results matches well with the values represented in Figure 11b. In all cases maximum response occurs at $U_{r}=8$. Even though the response amplitude is high in the range $U_{r}=4-12$, as it deviates away from $U_{r}=8$, amplitude decreases. A similar result was reported by [19] is represented in the modified Griffin's plot.

The numerical simulation could successfully reproduce the response behavior depicted by the lower branch of response. Figure $11 \mathrm{~b}$ shows the response of a cylinder of mass ratio $m^{*}=2.4$ which is comparable with the present study.

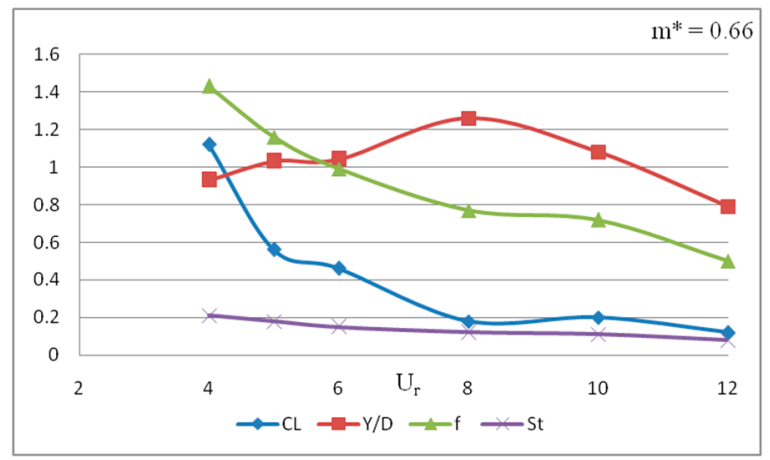

(a)

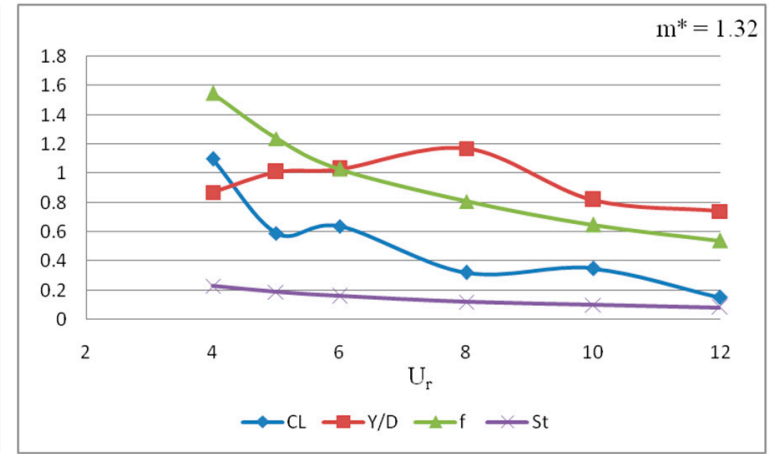

(b)

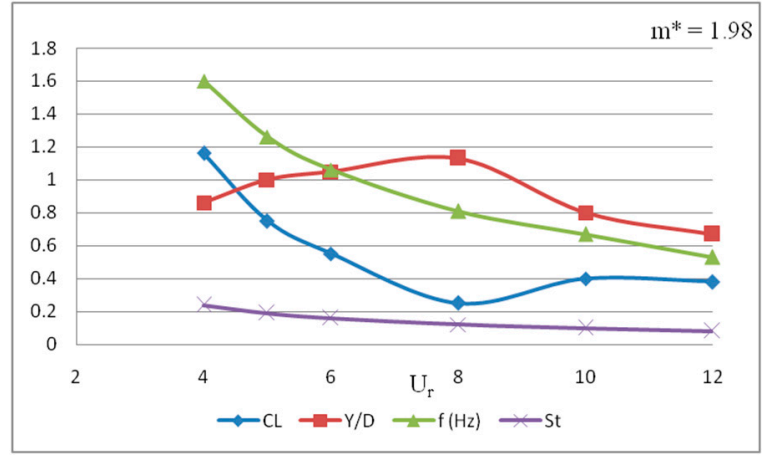

(c)

Figure 10. Effect of $U_{r}$ on various hydrodynamic and structural parameters for VIV of a cylinder (a) $m^{*}=0.66$; (b) $m^{*}=1.32 ;$ (c) $m^{*}=1.98$. 


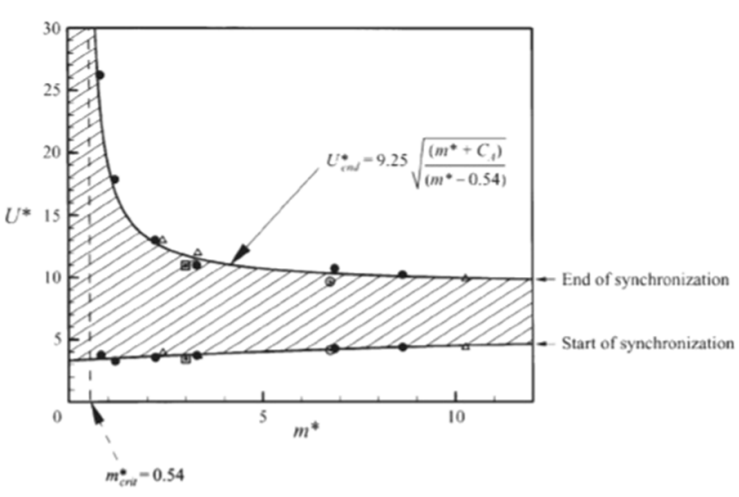

(a)

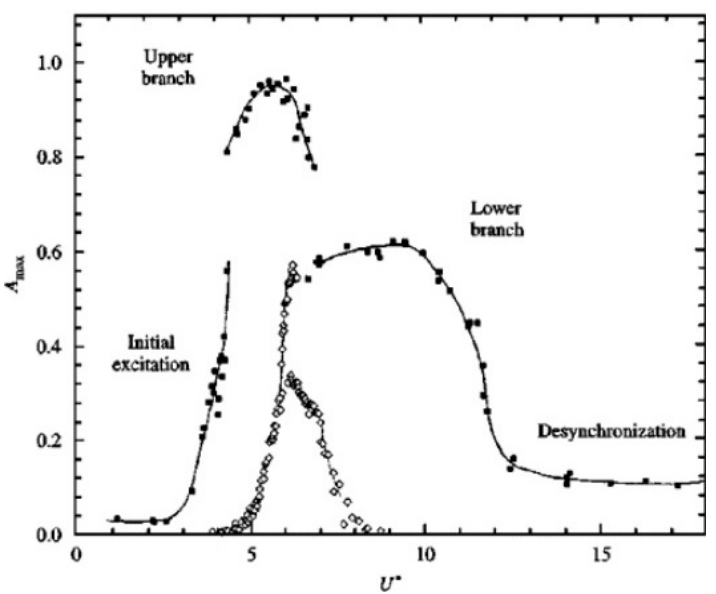

(b)

Figure 11. (a) Relationship between $U_{r}$ and $m^{*}$ presented by Williamson and Govardhan [23]; (b) maximum response amplitudes $A_{\max }$ as functions of the reduced velocity $U_{r}$ for $m^{*}=2.4$ and $m^{*}=248$ [19].

It was previously observed that $2 \mathrm{P}$ mode shedding is the reason for synchronized response [20]. Since detailed analysis of physics of the flow is not in the scope of this paper, shedding pattern is not further discussed. A representation of $2 \mathrm{P}$ mode of vortex shedding in the synchronization range of $U_{r}$ is shown in Figure 12. A detailed study to analyze this phenomenon is pending. Shedding characteristics at different $m^{*}$ and $k$ are listed in Table 4.

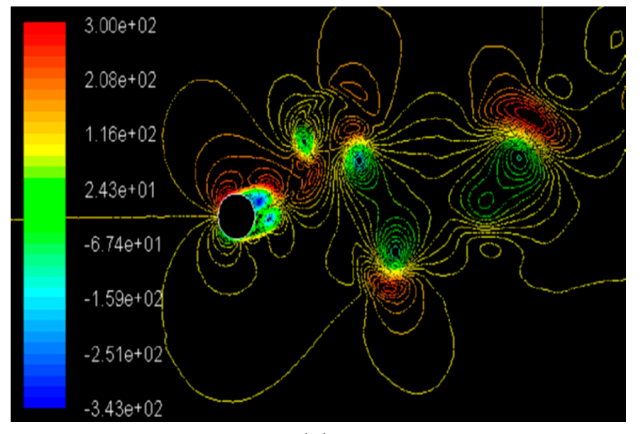

(a)

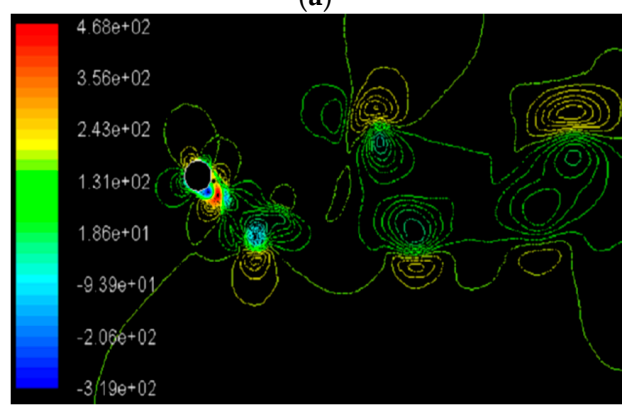

(c)

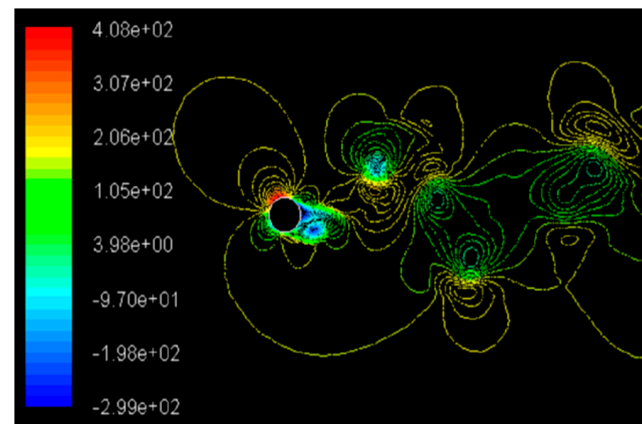

(b)

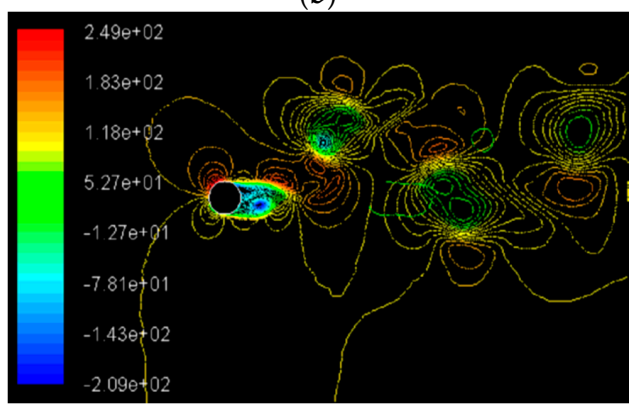

(d)

Figure 12. Cont. 


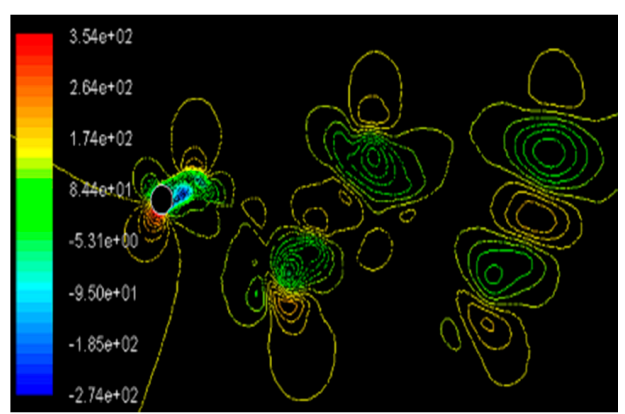

(e)

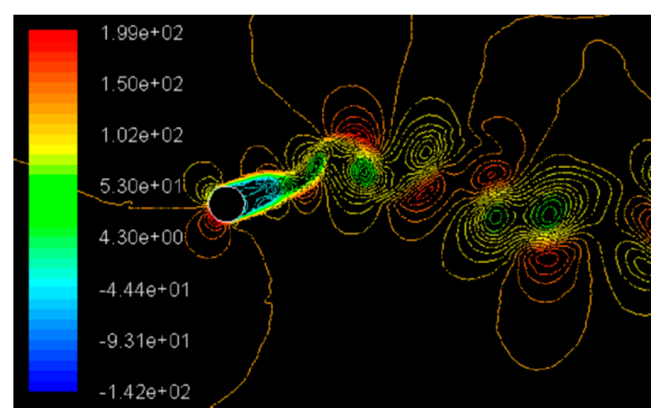

(f)

Figure 12. Representation of pressure $\left(\mathrm{N} / \mathrm{m}^{2}\right)$ contours in $2 \mathrm{P}$ mode of vortex shedding for $m^{*}=0.66$ (a) $U_{r}=4 ;$ (b) $U_{r}=5$; (c) $U_{r}=6$; (d) $U_{r}=8$; (e) $U_{r}=10$; (f) $U_{r}=12$.

Table 4. Hydrodynamic and structural response characteristics for $m^{*}=0.66,1.32$, and 1.98 .

\begin{tabular}{|c|c|c|c|c|c|c|c|}
\hline Mass Ratio, $m^{*}$ & $U_{r}$ & $C_{L}$ & $Y / D$ & $f_{v}(\mathrm{~Hz})$ & $\eta$ & $S t$ & Shedding Characteristics \\
\hline \multirow{6}{*}{$m^{*}=1.98$} & 4 & 1.16 & 0.86 & 1.6 & 1.04 & 0.24 & $\begin{array}{l}2 \mathrm{P} \\
\text { Lift force oscillates about zero value once during one } \\
\text { time period of oscillation of the cylinder. }\end{array}$ \\
\hline & 5 & 0.75 & 1.0 & 1.26 & 1.04 & 0.19 & $\begin{array}{l}\text { 2P } \\
\text { Lift force oscillates twice during one time period of } \\
\text { oscillation of the cylinder. }\end{array}$ \\
\hline & 6 & 0.55 & 1.05 & 1.06 & 1.03 & 0.16 & $\begin{array}{l}\text { 2P } \\
\text { Lift force oscillates twice during one time period of } \\
\text { oscillation of the cylinder. }\end{array}$ \\
\hline & 8 & 0.25 & 1.13 & 0.81 & 1.01 & 0.12 & $\begin{array}{l}\text { 2P } \\
\text { Lift force oscillates thrice during one time period of } \\
\text { oscillation of the cylinder. }\end{array}$ \\
\hline & 10 & 0.4 & 0.8 & 0.67 & 0.98 & 0.1 & $\begin{array}{l}\text { 2P } \\
\text { Lift force oscillates thrice during one time period of } \\
\text { oscillation of the cylinder. } \\
\text { Beat phenomenon is observed with time period } 10.53 \mathrm{~s} \text {. }\end{array}$ \\
\hline & 12 & 0.38 & 0.67 & 0.53 & 1.04 & 0.08 & $\begin{array}{l}\text { 2P } \\
\begin{array}{l}\text { Lift force oscillates } 3.5 \text { times during one time period of } \\
\text { oscillation of the cylinder. }\end{array}\end{array}$ \\
\hline \multirow{6}{*}{$m^{*}=1.32$} & 4 & 1.1 & 0.87 & 1.55 & 1.07 & 0.23 & $\begin{array}{l}2 \mathrm{P} \\
\text { Lift force oscillates about zero value once during one } \\
\text { time period of oscillation of the cylinder. }\end{array}$ \\
\hline & 5 & 0.59 & 1.01 & 1.24 & 1.06 & 0.19 & $\begin{array}{l}\text { 2P } \\
\text { Lift force oscillates twice during one time period of } \\
\text { oscillation of the cylinder. }\end{array}$ \\
\hline & 6 & 0.64 & 1.03 & 1.03 & 1.06 & 0.16 & $\begin{array}{l}2 \mathrm{P} \\
\text { Lift force oscillates twice during one time period of } \\
\text { oscillation of the cylinder. }\end{array}$ \\
\hline & 8 & 0.32 & 1.17 & 0.81 & 1.01 & 0.12 & $\begin{array}{l}\text { 2P } \\
\text { Lift force oscillates thrice during one time period of } \\
\text { oscillation of the cylinder. }\end{array}$ \\
\hline & 10 & 0.35 & 0.82 & 0.65 & 1.02 & 0.1 & $\begin{array}{l}\text { 2P } \\
\begin{array}{l}\text { Lift force oscillates thrice during one time period of } \\
\text { oscillation of the cylinder. }\end{array} \\
\text { Beat phenomenon is observed with time period } 10.42 \mathrm{~s} \text {. }\end{array}$ \\
\hline & 12 & 0.15 & 0.74 & 0.54 & 1.01 & 0.082 & $\begin{array}{l}\text { 2P } \\
\begin{array}{l}\text { Lift force oscillates } 3.5 \text { times during one time period of } \\
\text { oscillation of the cylinder. }\end{array}\end{array}$ \\
\hline \multirow{6}{*}{$m^{*}=0.66$} & 4 & 1.12 & 0.93 & 1.43 & 1.14 & 0.21 & $\begin{array}{l}2 \mathrm{P} \\
\text { Lift force oscillates about zero value once during one } \\
\text { time period of oscillation of the cylinder. }\end{array}$ \\
\hline & 5 & 0.56 & 1.03 & 1.16 & 1.13 & 0.18 & $\begin{array}{l}\text { 2P } \\
\text { Lift force oscillates twice during one time period of } \\
\text { oscillation of the cylinder. }\end{array}$ \\
\hline & 6 & 0.46 & 1.04 & 0.99 & 1.10 & 0.15 & $\begin{array}{l}2 \mathrm{P} \\
\text { Lift force oscillates twice during one time period of } \\
\text { oscillation of the cylinder. }\end{array}$ \\
\hline & 8 & 0.18 & 1.26 & 0.77 & 1.06 & 0.12 & $\begin{array}{l}\text { 2P } \\
\begin{array}{l}\text { Lift force oscillates thrice during one time period of } \\
\text { oscillation of the cylinder. }\end{array} \\
\text { Beat phenomenon is observed with time period } 7.84 \mathrm{~s} .\end{array}$ \\
\hline & 10 & 0.2 & 1.08 & 0.72 & 1.1 & 0.11 & $\begin{array}{l}\text { 2P } \\
\text { Lift force oscillates thrice during one time period of } \\
\text { oscillation of the cylinder. } \\
\text { No beat is observed. }\end{array}$ \\
\hline & 12 & 0.12 & 0.79 & 0.5 & 1.1 & 0.08 & $\begin{array}{l}\text { 2P } \\
\text { Lift force oscillates } 3.5 \text { times during one time period of } \\
\text { oscillation of the cylinder. }\end{array}$ \\
\hline
\end{tabular}


Energy possessed by a spring mass system undergoing oscillation can be represented as the sum of its kinetic and potential energies as given by Equation (26).

$$
E=\frac{1}{2} m \dot{Y}^{2}+\frac{1}{2} k Y^{2}
$$

When the position of the mass corresponds to maximum amplitude, the entire kinetic energy of the system will be converted into potential energy and Equation (26) reduces to

$$
E=\frac{1}{2} k Y^{2}
$$

At zero amplitude position of the mass, the entire potential energy is converted into kinetic energy. Since the total energy of the system is conserved energy balance can be written as Equation (28).

$$
E=\frac{1}{2} k Y^{2}=\frac{1}{2} m \dot{Y}^{2}
$$

Hence the maximum possible velocity with which the system oscillates can be expressed as

$$
\dot{Y}=\sqrt{\frac{k}{m}} Y
$$

Which may be also represented in non-dimensional form represented by Equation (30)

$$
\dot{Y}=\sqrt{\frac{D k^{*} g}{L / D}} \frac{Y}{D}
$$

For the optimum condition proven numerically, $V_{\max }$ is obtained as $0.5 \mathrm{~m} / \mathrm{s}$. Power associated with the oscillatory motion can be expressed by Equation (31)

Maximum velocity has been calculated using the above expression and a comparison of average power output estimated is represented in Table 5. Even though the maximum amplitude of oscillation is obtained for each $m^{*}$ at $U_{r}=8$, power output is maximum at $U_{r}=4$, suggesting the best

\begin{tabular}{|c|c|c|c|c|c|c|c|}
\hline$R e$ & $m^{*}$ & $u_{r}$ & $k^{*}$ & $C_{L}$ & $Y / D$ & $\dot{Y}$ & $P_{a v g}(W)$ \\
\hline \multirow{18}{*}{$3.8 \times 10^{4}$} & \multirow{6}{*}{0.66} & 4 & 11.17 & 1.12 & 0.93 & 0.74 & 7.90 \\
\hline & & 5 & 6.9 & 0.56 & 1.03 & 0.65 & 3.44 \\
\hline & & 6 & 4.81 & 0.46 & 1.04 & 0.54 & 2.38 \\
\hline & & 8 & 2.7 & 0.18 & 1.26 & 0.49 & 0.85 \\
\hline & & 10 & 1.73 & 0.2 & 1.08 & 0.34 & 0.64 \\
\hline & & 12 & 1.21 & 0.12 & 0.79 & 0.21 & 0.24 \\
\hline & \multirow{6}{*}{1.32} & 4 & 11.17 & 1.1 & 0.87 & 0.69 & 7.26 \\
\hline & & 5 & 6.9 & 0.59 & 1.01 & 0.63 & 3.55 \\
\hline & & 6 & 4.81 & 0.64 & 1.03 & 0.54 & 3.28 \\
\hline & & 8 & 2.7 & 0.32 & 1.17 & 0.46 & 1.40 \\
\hline & & 10 & 1.73 & 0.35 & 0.82 & 0.26 & 0.86 \\
\hline & & 12 & 1.21 & 0.15 & 0.74 & 0.19 & 0.28 \\
\hline & \multirow{6}{*}{1.98} & 4 & 11.17 & 1.16 & 0.86 & 0.69 & 7.57 \\
\hline & & 5 & 6.9 & 0.75 & 1 & 0.63 & 4.47 \\
\hline & & 6 & 4.81 & 0.55 & 1.05 & 0.55 & 2.87 \\
\hline & & 8 & 2.7 & 0.25 & 1.13 & 0.44 & 1.05 \\
\hline & & 10 & 1.73 & 0.4 & 0.8 & 0.25 & 0.95 \\
\hline & & 12 & 1.21 & 0.38 & 0.67 & 0.18 & 0.64 \\
\hline
\end{tabular}
operating conditions.

$$
P_{\text {avg }}=F_{L} \dot{Y}=C_{L} \frac{1}{2} \rho A V^{2} \dot{Y}
$$

Table 5. Cylinder velocity and calculated average power for different configurations. 


\section{Field Test Validation of Numerical Results}

The HVPG module with specifications as given in Table 1 has been used for a field test in the Palissery irrigation canal. The flow velocity in the canal was measured to be $0.5 \mathrm{~m} / \mathrm{s}$. The model has been tested for different $k^{*}$ values. Cylinder displacement was measured by attaching a pantograph to the side vanes. As the cylinder oscillates, the marking pencil attached to the spring side of the module marks its impression on the paper attached on the vane side. The results are tabulated in Table 6. $U_{r}=4,8$, and 12 , but the effect of $k^{*}$ is observed to follow the same trend as predicted numerically. An average deviation of $30 \%$ is observed between numerical method and field tests. Over prediction of the response amplitude by the numerical method may be attributed to not accounting for structural damping. Also, friction in the sliding parts of the guide vane contributes to lowering the response. Components of the HVPG and field test set up are shown in Figure 13.

Table 6. Results of field test conducted at Palissery irrigation canal.

\begin{tabular}{ccccccc}
\hline $\boldsymbol{R} \boldsymbol{e}$ & $\boldsymbol{m}^{*}$ & $\boldsymbol{U}_{\boldsymbol{r}}$ & $\boldsymbol{k}^{*}$ & $\boldsymbol{Y}_{\text {field }}(\mathrm{cm})$ & $\boldsymbol{Y} / D_{\text {fileld }}$ & $\boldsymbol{Y} / \mathrm{D}_{\text {numerical }}$ \\
\hline \multirow{3}{*}{$3.8 \times 10^{4}$} & & 4 & 11.17 & 5.5 & 0.72 & 0.93 \\
& \multirow{2}{*}{0.66} & 8 & 2.7 & 7.5 & 0.98 & 1.26 \\
& & 12 & 1.21 & 4.5 & 0.59 & 0.79 \\
\hline
\end{tabular}

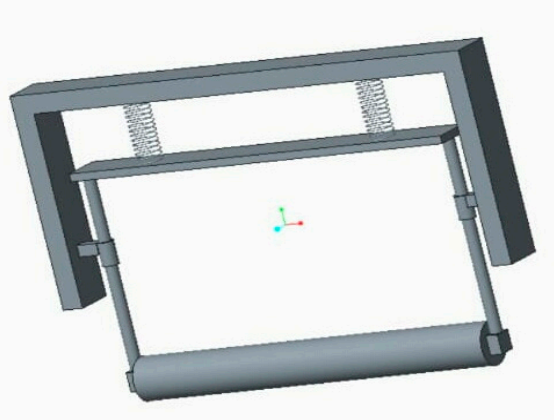

(a)

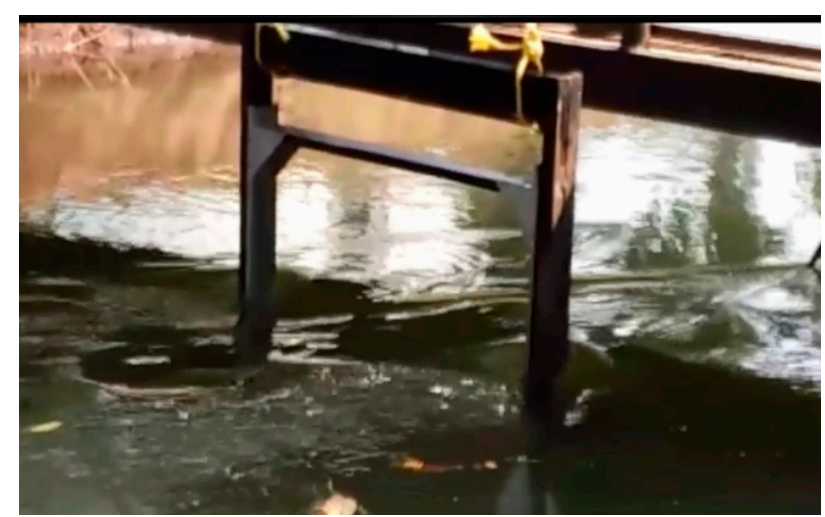

(b)

Figure 13. (a) Model of HVPG; (b) field test at Palissery irrigation canal for $m^{*}=0.66$ and $U_{r}=4,8$, and 12

\section{Conclusions}

Extensive research carried out to understand and interpret the intrinsic vortex shedding phenomenon has brought out several correlations to estimate response amplitude $Y / D$ [32], but most of these expressions define the non-dimensional oscillation amplitude $Y / D$ as a function of complex parameters like the Skop-Griffin parameter [14]. The present study is an effort to understand the hydrodynamic response of the cylinder from a designers' perspective by considering the effect of tangible system parameters only. Optimum response is obtained at $m^{*}=0.66$ and $U_{r}=8$, but optimum estimated power output for the same mass ratio is obtained at $U_{r}=4$. It is observed that maximum power output can be derived from an HVPG operating at low mass ratio and in the lowest regime of reduced velocity in the synchronization range irrespective of amplitude of cylinder response. The reduced power output at $U_{r}=8$ is due to the lower value of $C_{L}$ which in turn is due to several oscillations of lift force within one time period of oscillation of the cylinder. The true values of frequency ratio, $\eta$ obtained from the simulations are indicative of synchronized response for the range of $U_{r}$ considered. Hence it is observed that the developed numerical method could successfully simulate the flow around an oscillating cylinder. The numerical method developed is capable of predicting the trend of variation of $Y / D$ which is verified using the results of field test. At $m^{*}=0.66$ where maximum response is observed, $\eta$ significantly exceeds over unity compared to the higher mass ratio cases. It can 
be concluded that maximum amplitude of response is observed at mass ratios corresponding to $\eta$ values greater than unity rather than at unity $\eta$ values. Even though hydrodynamic lift force acting on the cylinder is proportional to the incoming flow velocity, $C_{L}$ strongly depends on the natural frequency of the oscillating system and the shedding pattern. Response amplitude of the cylinder also depends on stiffness ratio $k^{*}$ and in turn on natural frequency of the oscillating system $f_{n}$. Response amplitude increases with decreasing mass ratio and the range of $U_{r}$ over which the response is high (resonance) widens. Occurrence of beat phenomenon also depends on $m^{*}$ and $k^{*}$ and the relationship is more pronounced at lower values of $m^{*}$. The mode of vortex shedding depends only on $U_{r}$ at a constant flow velocity and is independent of $m^{*}$. On the whole, the work provides strong design inputs to the construction of the envisaged HVPG model.

\section{Scope for Future Research}

The discussions and conclusions from Sections 7 and 8 respectively indicate an immense scope for future research. Authors are in the process of improving the present design by incorporating structural damping in the studies. Inclusion of a greater number of rollers in the guide vanes is also being aimed at for eliminating the friction between sliding parts. The influence of these additional parameters in the equation of motion can reduce the errors in the subsequent numerical prediction, leading the design parameters closer to those of practical values. Improvised designs are also being devised for minimizing the transmission losses during power generation.

Author Contributions: Conceptualization, V.C., S.M., and S.J.; Data curation, V.C. and S.J.; Formal analysis, V.C. and S.J.; Funding acquisition, S.J. and V.M.; Investigation, V.C.; Methodology, S.M. and S.J.; Project administration, S.M. and S.J.; Resources, S.J. and V.M.; Software, V.M.; Supervision, S.M. and S.J.; Validation, V.C. and S.J.; Visualization, V.C. and S.J.; Writing-original draft, V.C. and S.J.; Writing-review \& editing, V.C., S.J., and V.M.

Funding: This research was partially funded by Energy Management Center, Government of Kerala, India specifically for the development of Hydro Vortex Power Generator module with grant no. EMC/ET\&R/18/R\&D/SCMS/01.

Acknowledgments: The authors would like to thank the management and principal of SCMS School of Engineering and Technology for all the support offered for the research and development. The authors would also like to thank Energy Management Center, Government of Kerala for all the support.

Conflicts of Interest: The authors declare no conflict of interest.

\section{References}

1. Gerrard, J.H. The mechanics of the formation region of vortices behind bluff bodies. J. Fluid Mech. 1966, 25, 401-413. [CrossRef]

2. Gao, Y.; Fu, S.; Xiong, Y.; Zhao, Y.; Liu, L. Experimental study on response performance of vortex-induced vibration on a flexible cylinder. Ships Offshore Struct. 2016, 12, 116-134. [CrossRef]

3. Bimbato, A.M.; Pereira, L.A.; Hirata, M.H. Suppression of vortex shedding on a bluff body. J. Wind Eng. Ind. Aerodyn. 2013, 122, 16-18. [CrossRef]

4. Bernitsas, M.; Raghavan, K.; Ben-Simon, Y.; Garcia, E. VIVACE (Vortex Induced Vibration Aquatic Clean Energy): A New Concept in Generation of Clean and Renewable Energy from Fluid Flow. J. Offshore Mech. Arct. Eng. 2006, 130, 041101. [CrossRef]

5. An, X.; Song, B.; Tian, W.; Ma, C. Design and CFD Simulations of a Vortex-Induced Piezoelectric Energy Conve rter (VIPEC) for Underwater Environment. Energies 2018, 11, 330. [CrossRef]

6. Janardhanan, S.; Chandran, V.; Varghese, C.; Achuth, D.; Devassy, D.; Mathews, D.C. Hydro vortex power generator design and construction. In Proceedings of the Kerala Technological CONGRESS, KETCON 2018-Human Computer Interface, Thrissur, India, 24 February 2018.

7. Griffin, O.M. Vortex-Excited Cross-Flow Vibrations of a Single Cylindrical Tube. ASME J. Press. Vessel Technol. 1980, 102, 158-166. [CrossRef]

8. Khalak, A.; Williamson, C.H.K. Dynamics of a hydroelastic cylinder with very low mass and damping. J. Fluids Struct. 1996, 10, 455-472. [CrossRef] 
9. Narendran, K.; Murali, K.; Sundar, V. Vortex-induced vibrations of elastically mounted circular cylinder at Re of the O(105). J. Fluids Struct. 2015, 54, 503-521. [CrossRef]

10. Govardhan, R.; Williamson, C.H.K. Defining the 'modified Griffin plot' in vortex-induced vibration: Revealing the effect of Reynolds number using controlled damping. J. Fluid Mech. 2006, 561, 147-180. [CrossRef]

11. Bernitsas, M. Out of the Vortex. Mech. Eng. 2010, 132, 22-27. [CrossRef]

12. Tian, W.; Mao, Z.; Zhao, F. Design and Numerical Simulations of a Flow Induced Vibration Energy Converter for Underwater Mooring Platforms. Energies 2017, 10, 1427. [CrossRef]

13. Khan, N.B.; Ibrahim, Z.; Tuan, L.; Javed, M.F.; Jameel, M. Numerical investigation of the vortex-induced vibration of an elastically mounted circular cylinder at high Reynolds number $(\operatorname{Re}=104)$ and low mass ratio using the RANS code. PLoS ONE 2017, 12, e0185832. [CrossRef] [PubMed]

14. Williamson, C.H.K.; Govardhan, R. Vortex induced vibrations. Annu. Rev. Fluid Mech. 2004, 36, $413-455$. [CrossRef]

15. Achenbach, E.; Heinecke, E. On Vortex Shedding from Smooth and Rough Cylinders in the Range of Reynolds Numbers $6 \times 103$ to $5 \times 106$. J. Fluid Mech. 1981, 109, 239-251. [CrossRef]

16. Blevins, R.D. Flow-Induced Vibration, 2nd ed.; Van Nostrand Reinhold: New York, NY, USA, 1990; pp. 163-164, ISBN 1-57524-183-8.

17. Gabbai, R.D.; Benaroya, H. An overview of modeling and experiments of vortex-induced vibration of circular cylinders. J. Sound Vib. 2005, 282, 575-616. [CrossRef]

18. Anagnostopoulos, P.W.; Bearman, P.W. Response characteristics of a vortex exited cylinder at low Reynolds number. J. Fluids Struct. 1992, 6, 39-50. [CrossRef]

19. Khalak, A.; Williamson, C.H.K. Investigation of the relative effects of mass and damping in vortex induced vibration of a circular cylinder. J. Wind Eng. Ind. Aerodyn. 1997, 69-71, 341-350. [CrossRef]

20. IcemCfd, A. 12.0 User's Ma; Ansys Inc.: Canonsburg, PA, USA, 2009; Volume 5.

21. Fluent, A. 12.0 Theory Guide; Ansys Inc.: Canonsburg, PA, USA, 2009; Volume 5.

22. Menter, F.R. Two-equation eddy-viscosity turbulence models for engineering applications. AIAA J. 1994, 32, 1598-1605. [CrossRef]

23. Anton, G. Analysis of Vortex-Induced Vibration of Risers. Master's Thesis, Applied Mechanics, Chalmers University of Technology, Gothenburg, Sweden, 2012.

24. Vandiver, J.K. Damping parameters for flow-induced vibration. J. Fluids Struct. 2012, 35, 105-119. [CrossRef]

25. Schlichting, H. Boundary Layer Theory, 8th ed.; McGraw-Hill Book Company: New York, NY, USA, 1979; ISBN 13 978-3540662709.

26. Iaccarino, G.; Mishra, A.A.; Ghili, S. Eigenspace perturbations for uncertainty estimation of single-point turbulence closures. Phys. Rev. Fluids 2017, 2, 024605. [CrossRef]

27. Mishra, A.A.; Gianluca, I. Uncertainty Estimation for Reynolds-Averaged Navier Stokes Predictions of High-Speed Aircraft Nozzle Jets. AIAA J. 2017, 55, 3999-4004. [CrossRef]

28. Govardhan, R.; Williamson, C.H.K. Modes of vortex formation and frequency response for a freely-vibrating cylinder. J. Fluid Mech. 2000, 420, 85-130. [CrossRef]

29. Chen, W.; Zhang, Q.; Li, H.; Hu, H. An experimental investigation on vortex induced vibration of a flexible inclined cable under a shear flow. J. Fluids Struct. 2015, 54, 297-311. [CrossRef]

30. Feng, C.C. The Measurements of Vortex Induced Effects in Flow Past a Stationary and Oscillating Circular and D-Section Cylinders. Master's Thesis, The University of British Columbia, Vancouver, BC, Canada, 1968.

31. Naudascher, E.; Rockwell, D. Flow Induced Vibration-An Engineering Guide; Dover Publications Inc.: Mineola, NY, USA, 2005; pp. 37-38, ISBN 13 978-0-486-44282-2.

32. Domal, V.; Sharma, R. An experimental study on vortex-induced vibration response of marine riser with and without semi-submersible. J. Eng. Marit. Environ. 2017, 232, 176-198. [CrossRef]

(C) 2018 by the authors. Licensee MDPI, Basel, Switzerland. This article is an open access article distributed under the terms and conditions of the Creative Commons Attribution (CC BY) license (http:/ / creativecommons.org/licenses/by/4.0/). 